Research paper

\title{
Analysis of the Fam181 gene family during mouse development reveals distinct strain-specific expression patterns, suggesting a role in nervous system development and function
}

\author{
Matthias Marks a,b, Tracie Pennimpede ${ }^{\mathrm{a}, 1}$, Lisette Lange ${ }^{\mathrm{a}, \mathrm{b}}$, Phillip Grote ${ }^{\mathrm{a}, 2}$, \\ Bernhard G. Herrmann ${ }^{\mathrm{a}, \mathrm{c}}$, Lars Wittler ${ }^{\mathrm{a}, *}$ \\ a Max Planck Institute for Molecular Genetics, Developmental Genetics Department, Ihnestraße 63-73, 14195 Berlin, Germany \\ ${ }^{\mathrm{b}}$ Free University Berlin, Dept. of Biology, Chemistry and Pharmacy, Takustrasse 3, 14195 Berlin, Germany \\ c Charité-University Medicine Berlin, Institute for Medical Genetics, Campus Benjamin Franklin, Hindenburgdamm 30, 12203 Berlin, Germany
}

\section{A R T I C L E I N F O}

\section{Article history:}

Received 2 February 2015

Received in revised form 5 June 2015

Accepted 9 September 2015

Available online 25 September 2015

\section{Keywords:}

Somitogenesis

Mouse embryogenesis

Neural development

Background specificity

Notch signaling

\begin{abstract}
A B S T R A C T
During somitogenesis differential gene expression can be observed for so-called cyclic genes, which display expression changes with a periodicity of $120 \mathrm{~min}$ in the mouse. In screens to identify novel cyclic genes in murine embryos, Fam $181 \mathrm{~b}$ was predicted to be an oscillating gene in the presomitic mesoderm (psm). This gene, and its closely related paralog Fam181a, belong to the thus far uncharacterized Fam181 gene family.

Here we describe the expression of Fam181b and Fam181a during murine embryonic development. In addition, we confirm oscillation of Fam181b in the psm in-phase with targets of, and regulated by, Notch signaling. Fam $181 \mathrm{~b}$ expression in the psm, as well as in the lateral plate mesoderm, was found to be affected by genetic background. We show that Fam $181 a$ and $b$ exhibit partially overlapping mRNA expression patterns, and encode for proteins containing highly-conserved motifs, which predominantly localize to the nucleus. A Fam $181 \mathrm{~b}$ lossof-function model was generated and found to result in no obvious phenotype.
\end{abstract}

(c) 2015 Elsevier B.V. All rights reserved.
Abbreviations: aa, amino acid; BAC, bacterial artificial chromosome; BLAST, basic local alignment search tool; bp, base pairs; cDNA, complementary DNA; cf, compare figure; $c$ hairy, chick hairy homolog 1; CMV-Cre, cytomegalovirus promoter driven Cre recombinase; $\mathrm{CO}_{2}$, carbon dioxide; $\mathrm{CpG}$-methylation, cytosine-guanine dinucleotidemethylation; DAPI, 4',6-diamidino-2-phenylindole; DIG, digoxigenin; Dkk1, dickkopf homo$\log$ 1; Dll1, delta-like 1; DMEM, Dulbecco's modified eagle medium; DNA, deoxyribonucleic acid; DTT, dithiothreitol; E, embryonic day; EDTA, ethylenediaminetetraacetic acid; EmGFP, emerald GFP; ES cell (ESC), embryonic stem cell; Fam181a, family with sequence similarity 181, member A; Fam181b, family with sequence similarity 181, member B; FCS, fetal calf serum; FGF, fibroblast growth factor; Fig., figure; FITC, fluorescein isothiocyanate; FlpE, flippase enhanced; GFP, green fluorescent protein; HEK293 cells, human embryonic kidney 293 cells; Hes1, hairy and enhancer of split 1; ID, identity; IgG, immunoglobulin G; kb, kilo bases; kDa, kilo dalton; Lfng, lunatic fringe; loxP, locus of crossover P1; lpm, lateral plate mesoderm; MAMEP, molecular anatomy of the mouse embryo project; MAPK, mitogen activated protein kinase; min, minutes; mM, millimolar; mRNA, messenger RNA; $\mu \mathrm{m}$, micrometer; $\mathrm{NaCl}$, sodium chloride; NP cells, neural progenitor cells; ORF, open reading frame; ov, otic vesicle; PBS, phosphate buffered saline; PCR, polymerase chain reaction; PFA, paraformaldehyde; Pmm2, phosphomannomutase 2; psm, presomitic mesoderm; qPCR, quantitative PCR; RNA, ribonucleic acid; RT-PCR, reverse transcriptasePCR; S, somite; T7, T7 RNA polymerase; TEAD4, TEA domain family member 4; TS, Theiler stage; U, unit; v/v, volume/volume; WISH, whole-mount in situ hybridization; Wnt, wingless-type MMTV integration site family; wt, wild type; YAP1, yes-associated protein 1.

* Corresponding author.

E-mail address: wittler@molgen.mpg.de (L. Wittler).

1 Present address: Queen's University, Division of Cancer Biology and Genetics, 10 Stuart St., K7L 3N6 Kingston, Canada.

2 Present address: Goethe University, Institute of Cardiovascular Regeneration, Theodor-Stern-Kai 7, 60590 Frankfurt am Main, Germany.

\section{Introduction}

During development of a complex multicellular organism, the cells which are being constantly generated require temporal and spatial instructions to ensure their correct positioning within the final body structure. Throughout embryonic development, cohorts of cells are instructed to collectively adjust their expression profiles, and thus commit and differentiate into tissues and organs. These changes in expression can occur at regularly spaced intervals - as in vertebrate segmentation. This process, called somitogenesis, leads to the bilateral generation of somites from the anterior end of the presomitic mesoderm (psm). The amount of time required for one somitogenic cycle is species-specific. In zebrafish, a somite pair buds off from the psm every $30 \mathrm{~min}$, in chicken every $90 \mathrm{~min}$, and every $120 \mathrm{~min}$ in the mouse. The molecular basis for somitogenesis is provided by morphogen gradients, which confer spatial information to the cells (Aulehla et al., 2003; Dubrulle et al., 2001; Del Corral et al., 2003; Moreno and Kintner, 2004), along with a molecular oscillator termed the segmentation clock. This ensures the correct spatiotemporal formation of somites (Cooke and Zeeman, 1976).

In 1997, Palmeirim and colleagues provided evidence for the existence of the segmentation clock on a molecular level. They showed that changes in the expression of the c-hairy1 gene in the psm were coordinated with somite formation in the developing chicken at $90 \mathrm{~min}$ 
intervals (Palmeirim et al., 1997). Since then, a number of additional "cycling genes" have been discovered in various species. These have been found to exclusively be targets of either the Notch-Dll (Palmeirim et al., 1997), the canonical Wnt (Aulehla et al., 2003), or the FGF-MAPK signaling pathways (Dequéant et al., 2006).

Recently, Dequéant et al. (2006) used a microarray-based screen of temporally-aligned mouse embryonic psm samples to perform a large-scale search for novel oscillating genes. Both in that study, and in a similar screen performed in our lab (P. Grote, L. Wittler, M. Werber, and B.G. Herrmann, unpublished data), the thus-far uncharacterized gene Fam181b (synonym A830059I20Rik) was identified as an oscillating transcript with a possible function during segmentation.

The intron-less Fam 181b gene is located on mouse chromosome 7 and is predicted to encode a protein with a length of 417 aa $(\sim 42 \mathrm{kDa})$. It has one paralog, Fam181a (synonym EG544888), located on mouse chromosome 12 , which encodes a protein of 292 aa $(\sim 32 \mathrm{kDa})$. In this study we analyze the expression patterns of Fam181a and Fam181b during murine embryonic development and in adult organs, and present initial investigations into the function of the gene family members, thus providing the first comprehensive characterization of the murine Fam181 gene family.

\section{Materials and methods}

\subsection{Whole-mount in situ hybridization and vibratome sectioning}

For whole-mount in situ hybridization (WISH), embryos were processed according to the protocol provided by the MAMEP database (http://mamep.molgen.mpg.de). The probe for Fam181b corresponds to nucleotides 882-1813 of NM_021427.2, and the probe for Fam181 a to nucleotides 606-1343 of NM_001195726.1. Probe templates were produced by PCR with a reverse primer containing a T7 site for antisense transcription. DIG-labeled probes were generated by in vitro transcription according to standard procedures, and staining was performed using BM Purple (Roche). Following the staining reaction, samples were postfixed in $4 \%$ PFA/PBS overnight. Some specimens were used to generate vibratome sections ( $35 \mu \mathrm{m}$ thickness) following a sucrose gradient and embedding in a glycerin/albumin matrix.

\subsection{In situ hybridization on paraffin sections}

Embryos were fixed in 4\% PFA/PBS overnight, processed into paraffin wax by standard procedures and sectioned using a microtome $(5 \mu \mathrm{M})$. In situ hybridization was performed on the sections according to the protocol from Chotteau-Lelièvre et al. (2006), with minor modifications. The staining reaction was performed using BM Purple (Roche). For each stage examined at least 3 sections from 2 different embryos were analyzed. There was no observed variation in the staining pattern, and figures show representative staining.

\subsection{Tail half cultures}

For tail half culture experiments, E9.5 mouse embryos were dissected into ice-cold PBS and their caudal ends bisected along the neural tube using a tungsten needle, leaving several somites anterior to the psm. After incubation of both halves for $30 \mathrm{~min}$ in DMEM/F12/10\% FCS at 37 ${ }^{\circ} \mathrm{C} / 7.5 \% \mathrm{CO}_{2}$, one half was fixed in $4 \% \mathrm{PFA} / \mathrm{PBS}$, while the second half was further incubated for $90 \mathrm{~min}$ or $120 \mathrm{~min}$ prior to fixation. Corresponding halves were then processed simultaneously for WISH as described above. For comparisons of gene expression at the same timepoint, both halves were immediately fixed after bisection.

\subsection{Generation of Fam181b-V5 knock-in and knock-out embryos}

To generate a knock-in vector, the genomic region containing the Fam $181 b$ transcript and a $2.6 \mathrm{~kb} 3^{\prime}$ homology arm were amplified by
PCR from the RP23-168D4 BAC (BACPAC Resources Center, Oakland, CA, USA). The $3^{\prime}$ homology arm contained a repeat of the last $139 \mathrm{bp}$ of the transcript at its $5^{\prime}$ end added by the PCR primer. The V5-tag was inserted at the $3^{\prime}$ end of the Fam 181 b ORF by fusion-PCR. Both modified transcript and homology arm were then inserted into the PL451 vector ((Pgk): Frt-Pgk-em7-Neo-Frt-loxP) (Liu et al., 2003) upstream of the floxed PGK-Neo cassette. For the knock-out vector, a $2.9 \mathrm{~kb}$ fragment upstream of the Fam $181 \mathrm{~b}$ transcriptional start site was amplified adding a loxP site to the $3^{\prime}$ end. This served as $5^{\prime}$ homology arm and was subcloned, together with the Fam $181 \mathrm{~b}$ transcript coding region and the 3' homology arm, into the PL451 vector upstream of the floxed PGK-Neo cassette. Linearized vector for either the knock-in or knockout constructs was then used for targeted integration into the Fam181b locus of G4 mouse embryonic stem (ES) cells (129S6/SvEv $\times$ C57BL/6 N background), and correct integration was verified by Southern blot. For the knock-in, the selection cassette was subsequently removed by transient transfection of positively targeted ESCs with a FlpE-containing expression plasmid. Negative selection and Southern blot verified loss of the cassette. Highly chimeric embryos were generated (70-80\% chimerism) by morula aggregation (Eakin and Hadjantonakis, 2006). To generate knock-out animals, chimeric F0 animals were directly crossed to $C M V$-Cre animals (C57BL/6 J background) for deletion of the transcript and the selection cassette. The offspring were then intercrossed, and the resulting embryos/animals used for analysis. All animal procedures were performed in ethical accordance with protocols set out by the Max Planck Institute for Molecular Genetics, with prior approval of the Berlin Animal Welfare Authorities (LAGeSo).

\subsection{Differentiation of ESCs along the neural lineage}

Murine G4 ESCs were grown under feeder-free conditions and subjected to in vitro differentiation into glutamatergic neurons according to the protocol established by Bibel et al. (2007). Samples were taken every 2 days after the formation of cellular aggregates.

\subsection{RNA extraction, $c D N A$ synthesis, and quantitative PCR}

For RNA extraction, samples were lysed in TRIzol® Reagent (Life Technologies). Total RNA was extracted using the RNeasy Plus Mini Kit (Qiagen) and transcribed into cDNA using the QuantiTect ${ }^{\circledR}$ Reverse Transcription Kit (Qiagen), both according to the manufacturer's protocols. For real-time quantitative PCR, cDNA and appropriate primer pairs were combined with GoTaq ${ }^{\circledR}$ qPCR Master Mix (Promega) and run on the StepOnePlus Real-Time PCR System (Life Technologies). Analysis was performed using either the StepOne software v2.3 (Life Technologies) or the $\Delta \Delta \mathrm{ct}$ method and q-gene (Muller et al., 2002). Pvalues were calculated using a one-tailed, paired Student's t-test. For semi-quantitative PCR (RT-PCR), cDNA was used in a standard PCR reaction with GoTaq ${ }^{\circledR}$ Flexi DNA polymerase (Promega). The following mouse-specific primers were used: Fam181a fwd: cctatcccgactaagccagc/Fam181a rev: gccaaaagagagagggctga; Fam181b fwd: cttcccagattgtgcgttgc/Fam181b rev: tctccagaggctggggtaaa; Oct4 fwd: tgttccegtcactgctctgg/Oct4 rev: ttgccttggctcacagcatc; Pax6 fwd: catggcaaacaacctgcctatg/Pax6 rev: gcacgagtatgaggaggtctgac; TrkB fwd: agcagccctggtatcagcta/TrkB rev: cttgatgttcttccgggtgt; Lfng fwd: ctgca ccattggctacattg/Lfng rev: tgctgcaggttctctaggtg; Pmm2 fwd: agggaaaggcc tcacgttct/Pmm2 rev: aataccgcttatcccatccttca; Gapdh fwd: tcaagaaggtggt gaagcag/Gapdh rev: accaccctgttgctgtagcc.

\subsection{Transient transfection, immunofluorescence, and immunoblotting}

Transient transfection of NIH3T3, HEK293, and C2C12 cells (ATCC Germany) was performed using Lipofectamine ${ }^{\mathrm{TM}} 2000$ reagent (Life Technologies) according to the manufacturer's instructions. Detection of proteins was performed using an $\alpha$-V5 primary antibody (Life Technologies; R960-25) at a 1:1000 dilution, or an $\alpha$-GFP antibody (Life 
Technologies; A11122) at a 1:500 dilution. Secondary antibodies used for immunofluorescence were $\alpha$-rabbit IgG-Alexa488 conjugated (Life Technologies; A11034), and $\alpha$-mouse IgG-Alexa546 conjugated (Life Technologies; A11030), both at 1:1000. Counterstaining was performed by incubation with FITC-phalloidin at 1:500 (Sigma; P5282), and slides were mounted using VECTASHIELD HardSet Mounting Medium with DAPI (Vector Laboratories).

Whole embryo lysates were prepared using TOPEX buffer (300 mM $\mathrm{NaCl} / 50$ mM Tris-HCL pH 7.5/0.5\% Triton X-100/1 mM DTT/1 × complete EDTA-free protease inhibitors (Roche) plus $33.33 \mathrm{U} / \mathrm{ml}$ Benzonase ${ }^{\circledR}$ (Sigma; E1014-25KU) at a ratio of 10:1 v/v of embryonic sample. Immunoblotting was performed using standard procedures with primary $\alpha$-V5 at 1:500, and $\alpha$-mouse Laminin B1 loading-control (Abcam ab16048) diluted 1:3500. Secondary antibodies used were $\alpha$-mouse or $\alpha$-rabbit HRP-linked IgG (Cell Signaling; 7076 and 70745), both at 1:2000. Chemiluminescence detection was performed using the Amersham ${ }^{\mathrm{TM}} \mathrm{ECL}^{\mathrm{TM}}$ Western blotting Detection Reagents (GE Healthcare) and images were acquired on a Fusion SL Vilber Lourmat device (Peqlab).

\subsection{Imaging}

For imaging of WISH-stained embryos, a MZ16A dissection microscope (Leica) fitted with an AxioCam MRc5 (Carl Zeiss MicroImaging) were used in combination with the AxioVision Software (Carl Zeiss Microlmaging). Vibratome sections were imaged using a Zeiss Observer.Z1 microscope with an AxioCam MRc (Carl Zeiss Microlmaging) and the AxioVision Software. Fluorescence microscopy was performed on an LSM710 laser-scanning microscope using the ZEN software (Carl Zeiss Microlmaging).

\subsection{Phylogenetic analyses}

Sequence alignment of human and murine FAM181 proteins was generated using CLC DNA workbench. Multiple sequence alignment of the selected vertebrate species was produced using Clustal Omega (Sievers et al., 2011) with default settings. This alignment was used as input for ClustalW version 2 (Larkin et al., 2007) to generate the phylogenetic tree. The distance correction was enabled by the software, while other settings remained default. Conversion of the Newick tree into an SVG tree was done using TreeVector (http://supfam.cs.bris.ac.uk/ TreeVector/index.html). Sequence identities were calculated using William Pearson's lalign program (http://www.ch.embnet.org/ software/LALIGN_form.html). The reference sequences used for the alignment and generation of the phylogenetic tree were: Alligator mississippiensis FAM181A XP_006278984.1; Anolis carolinensis FAM181A XP_003214448.1; A. carolinensis FAM181B XP_008106372.1; Bos taurus FAM181A XP_594106.4; B. taurus FAM181B NP_001094693.1; Danio rerio FAM181A XP_005169962.1; D. rerio FAM181B XP_005157544.1; Gallus gallus FAM181A XP_003641418.1; G. gallus FAM181B XP_ 004939010.1; Homo sapiens FAM181A NP_612353.3; H. sapiens FAM181B NP_787081.2; Macaca mulatta FAM181A gb|EHH28130.1; M. mulatta FAM181B NP_001180963.1; Monodelphis domestica FAM181A XP_001370835.2; M. domestica FAM181B XP_001377183.1; Mus musculus FAM181A NP_001182655.1; M. musculus FAM181B NP_ 067402.2; Nematostella vectensis predicted protein XP_001627460.1; Pan troglodytes FAM181A XP_001143456.2; P. troglodytes FAM181B XP_003313276.1; Xenopus tropicalis FAM181A gb|AAI35265.1; and X. tropicalis FAM181B XP_004912246.1.

\subsection{Mouse strains}

For the analysis of Fam181a and Fam181b expression patterns in embryos, and the characterization of Fam181b oscillation in the psm, the outbred CD1 and NMRI strains were used. For expression analysis of Fam181 genes in adult tissues, samples were taken from a 53 week-old CD1 female animal. The Dll1 transgenic mouse line (Hrabe de Angelis et al., 1997) was maintained at heterozygosity on a CD1 background. The outbred strains C57BL/6J and 129S2SvHsd were used for analysis of the background-dependency of Fam 181b expression. The Dkk1 mouse line (Mukhopadhyay et al., 2001) was maintained heterozygously on both C57BL/6J, and 129S2SvHsd backgrounds. Here we used Dkk1-/embryos resulting from intercrosses of both strains.

Mice were maintained in the animal facility of the Max Planck Institute for Molecular Genetics, Berlin, in accordance with international standards and protocols. Animal maintenance and all procedures performed on mice described here were performed in accordance with the German animal welfare act (Tierschutzgesetz, TSchG) and had prior approval from local authorities (LaGeSo).

\section{Results and discussion}

\subsection{The FAM181 protein family is highly conserved among vertebrates}

In order to classify the mouse Fam181 gene family in a phylogenetic context, we compared the predicted protein sequences of murine Fam181 genes with those from orthologous genes in other species. mFAM181A and mFAM181B show $46 \%$ similarity on the amino acid level, while multiple sequence alignment with their human homologs showed a similarity of about $77 \%$ for both orthologous pairs (Fig. 1A). Within all human and mouse sequences, we found 4 conserved domains (Fig. 1A, red and green boxed regions). To further analyze this conservation, we performed a protein-protein BLAST search with the mouse FAM181s using non-redundant sequences to identify putative orthologs by sequence similarity. This analysis illustrated that the FAM181 family is highly conserved among vertebrates. In most species the BLAST search revealed two proteins, one more similar to mFAM181A and the other to mFAM181B. Next, we performed a multiple sequence alignment for selected species, representing different taxa of vertebrates, which we used to generate a phylogenetic tree (Fig. 1B) using the starlet sea anemone $N$. vectensis as an outgroup. The existence of both paralogs was found among most vertebrate species. In addition, the tree confirmed the conservation of the FAM181 family along the vertebrate phylum, with a large portion of highly conserved aa within 3 of the 4 conserved boxes (Fig. 1A, orange lettered residues). The fourth box, containing a proline-rich stretch (Fig. 1A, green boxed region), was also found to be conserved, though the exact position and total number of proline residues varied between the species investigated. In the nonavian sauropsid A. mississippiensis (American alligator) only a FAM181A ortholog was recovered, likely due to its incomplete genome sequence (St John et al., 2012).

The three boxes of highly conserved residues and the proline-rich stretch might play a role in the function of these proteins. A structural homology search using the Phyre ${ }^{2}$ online tool (Kelley and Sternberg, 2009) showed a region of high similarity within FAM181B to a motif from the Hippo signaling effector yes-associated protein 1 (YAP1, Fold library IDs c3kysB and c3juaB), which is required for recognition by the DNA-binding protein TEAD4 (Chen et al., 2010; reveiwed in Pan, 2010). This region partially overlapped with the conserved box 3 (residues 209 to 231) and was also found by a BLAST search with the third conserved box against all annotated mouse proteins. However, the functional relevance of this finding remains to be evaluated.

\subsection{FAM181A/B proteins predominantly localize to the nucleus}

The FAM181 proteins were thus-far uncharacterized, aside from their grouping by sequence similarity. Since the Ensemble Genome Browser annotates the murine Fam $181 \mathrm{~b}$ gene as a pseudogene (ENSMUSG 00000051515) we wanted to examine whether FAM181B is expressed in vivo. Therefore, we integrated a C-terminal V5-tag into one of the endogenous Fam $181 b$ alleles in murine ESCs, which were then used to generate chimeric embryos (Fig. 2A). We were able to detect FAM181B-V5 with the expected size ( $42 \mathrm{kDa}$; Fig. 2B, V5-KI lane) by 
A Mus musculus FAM181B - ....... - MAVQAALLS SHPFIPFGFG GSADGLVSAF GSLDKGCCFE 39 Homo sapiens FAM181B ............. -MAVQAALLS THPFVPFGFG GSPDGLGGAF GALDKGCCFE 39

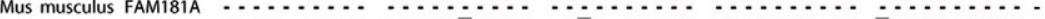
Homo sapiens FAM181A MPLEERRSSG ERNDAAPTNH RRPGEKRTST AKQVSSVPFL GAAGHQQSLP 50 Mus musculus FAM181B DDESGASAGA LLSGSEGGDV REATRDLLSE TDSASSNDIKL- ALDKPGKSKR 89 Homo sapiens FAM181B DDETGAPAGA LLSGAEGGDV REATRDLLS IDSASSN IKL ATLDKPGKSKA 89

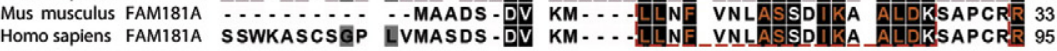
Mus musculus FAM181B KVNARTYLIK DTKRCSGLMG TAPPRPASPS AADAPAKRP - - -PGAPTVAT 136 Homo sapiens FAM181B KVNHRKYLAK QIKRCSGLMG AAPPGPPSPS AADTPAKRPL AAPSAPTVAA 139 Mus musculus FAM181A SVDHIRYLGK GLKRFSQKYS RLPRGLPGRV ÄEPHLQRGPE ERPGRPPLHP 83

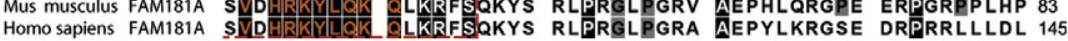
Mus musculus FAM181B PAHCKAAPRR EATQAAAAAS LQSRSLAALF DSLRHIPGGA ETAGGAEAVS 186 Homo sapiens FAM181B PAHGKAAPRR EASQAAAAAS LQSRSLAALF DSLRHVPGGA EPAGGEVAAP 189
Mus musculus FAM181A

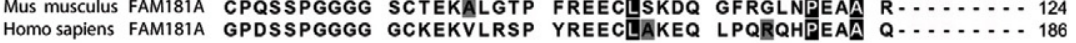
Mus musculus FAM181B VPGLGAAS - - -AVGDGAGTA VSSVAPGTRK VFLRARNLPF SFFTEPSRVG 233 Homo sapiens FAM181B AAGLGGAGTG GAGGDVAGPA GATAIPGARK VILAARNPP SFFTEPSRA 239

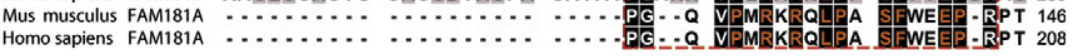
Mus musculus FAM181B CGGASGVPSG QGVSLGDLEK GAEAVEFFEL LAPDFGSGND SGVLMA-ADP 282 Homo sapiens FAM181B GGGCG.-PSG PDVSLGDLEK GAEAVEFFEL LGPDYGAGTE AAVLLA-AEP 286 Mus musculus FAM181A LSYPMGLEVG LAPREASLYE NKKNCKGLES LGPETAPLPM SPRVLADTEP 196 Homosapiens FAM181A HSYHVGLEGG LGPREGPPYE GKKNCKGLEP LGPETTLVSM SPRALAEKEP 258 Mus musculus FAM181B LDPFPAGATV LRGPLELESG PFEQP.AMVG NLLYPEPWNT PSCPQTKKPP 331 Homo sapiens FAM181B LDVFPAGASV LRGPPELEPG LFEPPPAVVG NLLYPEPWSV PGCSPTKKSP 336

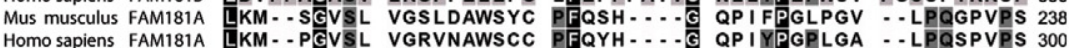
Mus musculus FAM181B VAGVRGgVTL NEPVRLEYPT ALDSPGGEDA PA-LSSFTPF FPDCALPPP: 379 Homosapiens FAM181B LTAPRGGLTL NEPLSPLYPA AADSPGGEDG RGHLASFAPF FPDCALPPPP 386 Mus musculus FAM181A LGLWRRSLAS PVDLAHFCK - GVDTPGPKVY RPVVLKPIPT KP.--AMPPPI 285

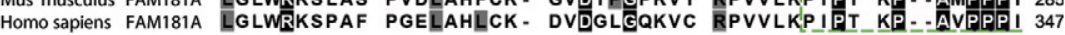

Mus musculus FAM181B --THQVSYDYS A-GYSRAVYP SLWRPDGVWE GASGEEGGHP D 417 Homo sapiens FAM181B PPHQVSYDYS A-GYSRTAYS SLWRSDGVWE GAPGEEGAHR D 426

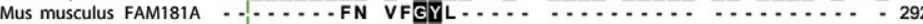

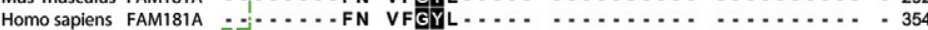

Conserved between:

$$
\begin{array}{ll}
\text { A }^{\text {human and murine Fam 181 B }} & \mathbf{A}^{\text {all } 4 \text { homologs }} \\
\mathbf{A}_{\text {(FAM 181 A or FAM 181 B) }}^{3 \text { out of } 4 \text { homologs }} & \mathbf{A}_{\text {phylogram species (except outgroup) }}^{\text {all } 4 \text { homologs+ }}
\end{array}
$$

B

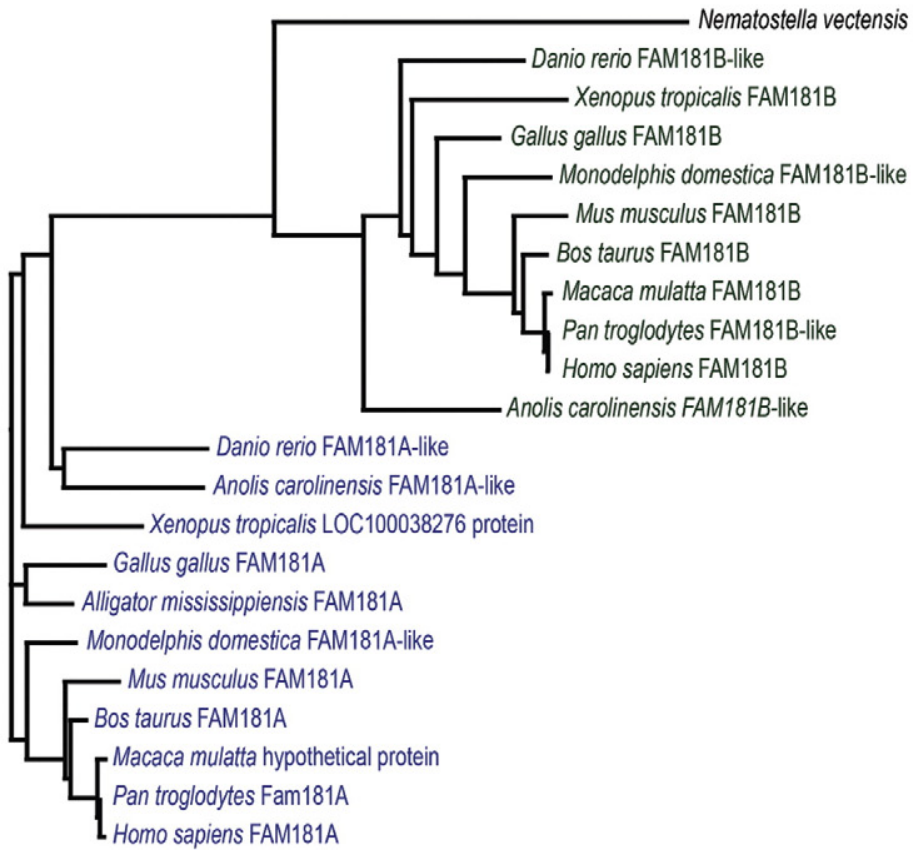

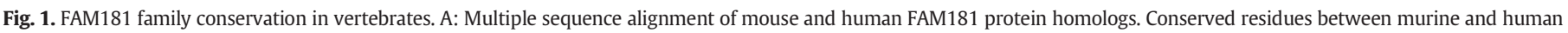

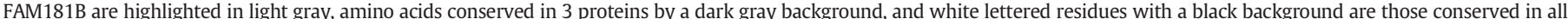

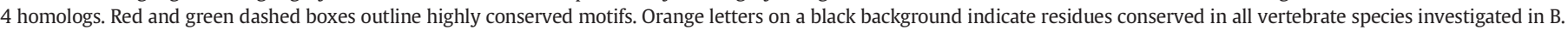

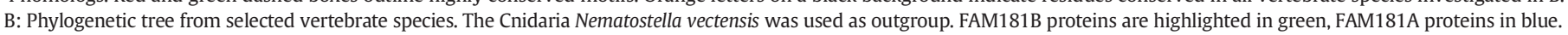

immunoblotting of whole embryo protein lysates from TS13-14 knock-in embryos, as compared to control embryo lysate. This provides strong evidence that Fam181b is a protein-coding gene with the predicted amino acid sequence.
We went on to determine the subcellular localization of FAM181B by transiently transfecting NIH3T3 cells with an expression construct encoding N-terminally V5-tagged FAM181B. These cells were analyzed by indirect immunofluorescence for V5, and counterstained with DAPI 
A

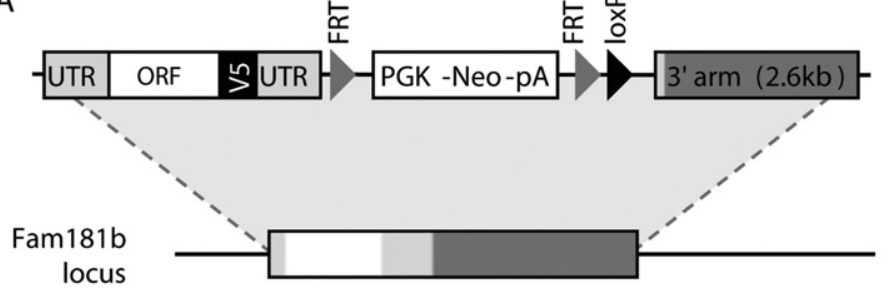

B

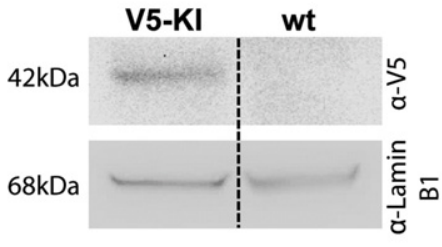

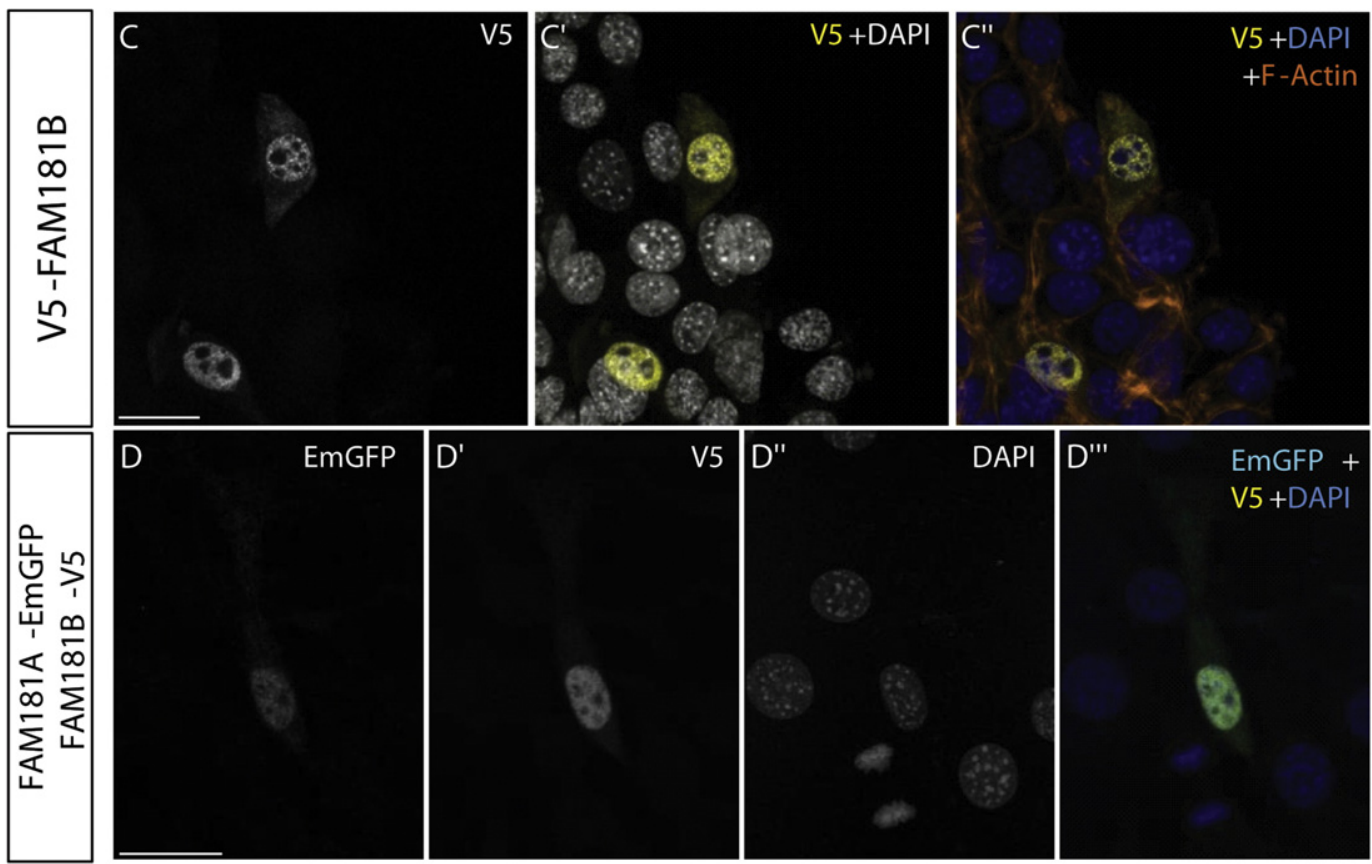

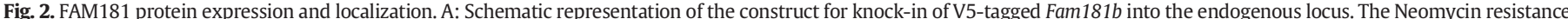

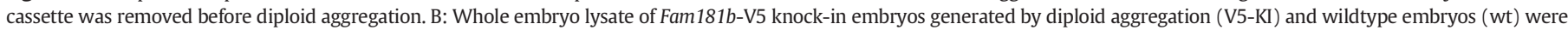

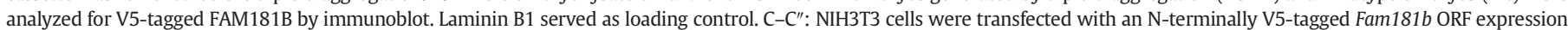

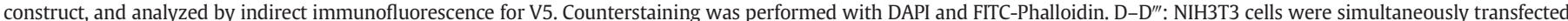

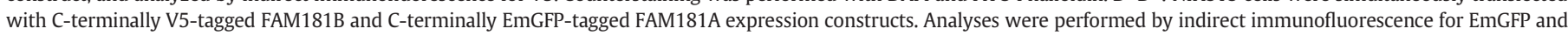
V5, and cells were counterstained with DAPI. Scale bar $=25 \mu \mathrm{m}$.

and FITC-phalloidin to visualize the nuclei and the F-actin cytoskeleton, respectively. Strong nuclear localization was observed for the tagged protein, with a much weaker signal in the cytoplasm (Fig. 2C- $\mathrm{C}^{\prime \prime}$ ). This localization was also observed when the V5-tag was located Cterminally (compare to Fig. 2D'), when a GFP-tag was used instead of V5, and also in HEK293 and C2C12 cells (data not shown).

Using the antibody HPA001603 from the Human Protein Atlas, hFAM181A was previously found to localize to nucleoli of U-2 OS human osteosarcoma cells, although an siRNA-mediated knock-down of Fam181a in the same study failed to validate this result (Stadler et al., 2012). In order to compare the localization of both homologs in our system, we co-transfected NIH3T3 cells with FAM181B-V5 and FAM181A-EmGFP expression constructs. Immunofluorescence with DAPI-counterstaining showed an enrichment in the nucleus with weaker speckles in the cytoplasm for FAM181A (Fig. 2D-D'"). Thus, although they lack a known nuclear localization signal, both murine FAM181 proteins localize to the nucleus in transiently-transfected cells. As previously mentioned, both proteins also share the conserved box 3 region with structural homology to the Yap-Tead4 interaction interface (see Fig. 1A). YAP requires TEAD proteins for its nuclear localization and to exert its function (Vassilev et al., 2001; Cao et al., 2008). However, deletion of box 3 from the FAM181B-V5 expression construct did not alter its nuclear localization in transiently-transfected NIH3T3 cells (data not shown). Thus, another domain within FAM181B, and likely also FAM181A, must be responsible for the subcellular localization. Further experiments are needed to validate the localization of endogenous proteins under physiological conditions and to address their molecular functions.

\subsection{Fam181b is dynamically expressed during embryonic development}

To investigate the expression of Fam $181 \mathrm{~b}$ during murine embryonic development, we performed an extensive analysis by whole-mount in situ hybridization (WISH) on wild type (wt) embryos between embryonic day (E) 6.5 and E12.5. We further analyzed the expression domains on a histological level by generating vibratome sections for some of the specimens or performing in situ hybridization on midsagittal paraffin sections at E14.5.

Fam181b transcripts first became detectable at E7.5 in the prospective headfold region of late allantoic bud stage embryos (Fig. 3A/A'). During headfold formation, this domain narrowed to a smaller area (Fig. 3B/B') which corresponds to the midbrain at later stages (Fig. 3C, white dashed line). At Theiler stage (TS) 12, Fam181b mRNA was detected in two further regions, one in the psm, and another in the rhombencephalon (Fig. 3C/ $\mathrm{C}^{\prime}$, black dashed line and red arrowhead, respectively). Both midbrain and psm expression were maintained during all stages of embryonic development investigated (Fig. 3C-H), while the rhombencephalic signal was undetectable after TS14 (Fig. 3D). In agreement with its identification in screens for oscillatory expressed genes in the psm (Dequéant et al. 2006 and our unpublished data), we observed that the anteroposterior extension of the Fam $181 \mathrm{~b}$ psm expression domain varied between specimens (also see Fig. 7A-A"), suggesting oscillation of Fam $181 \mathrm{~b}$ 

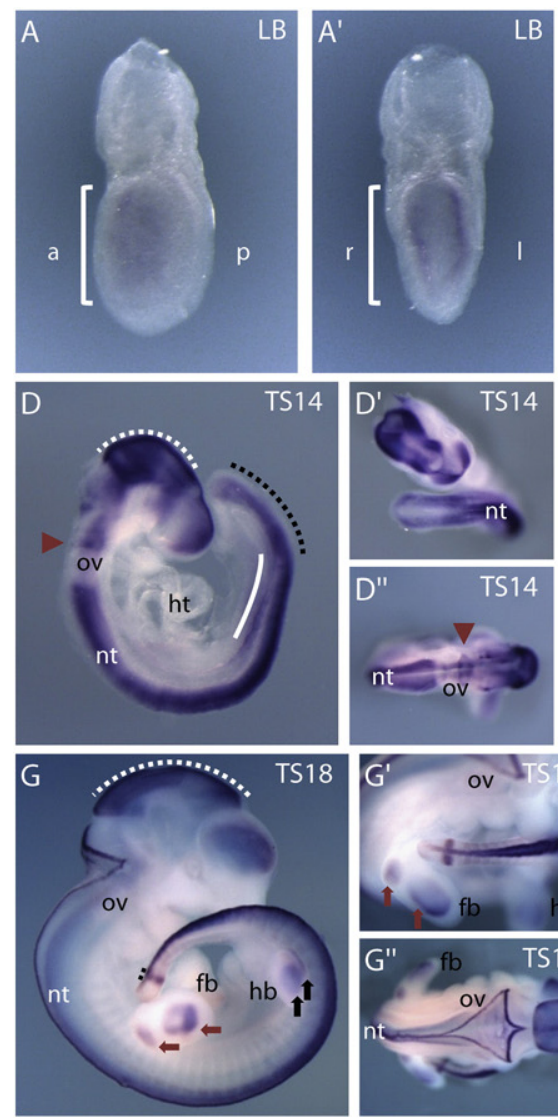
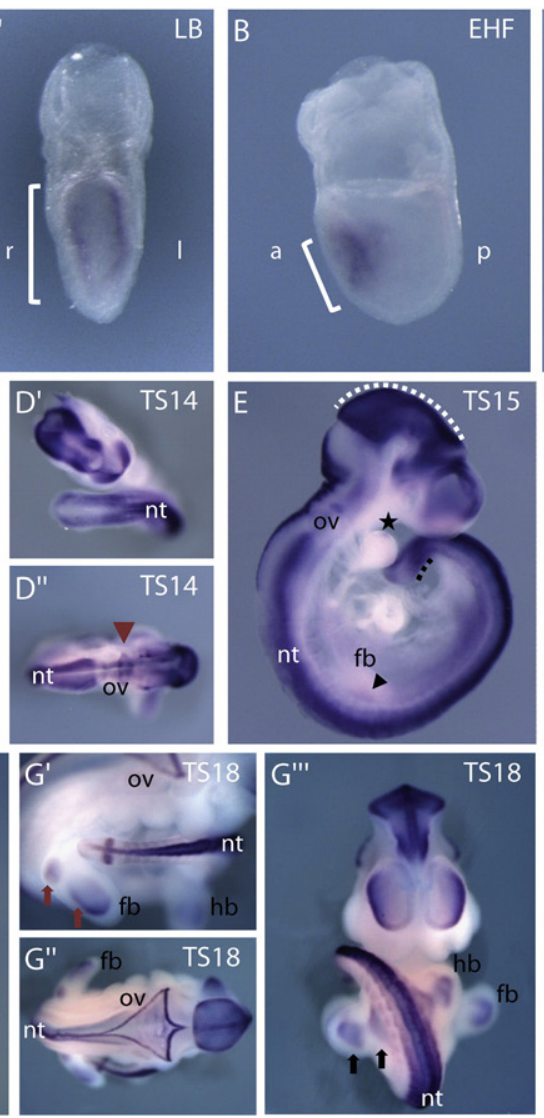
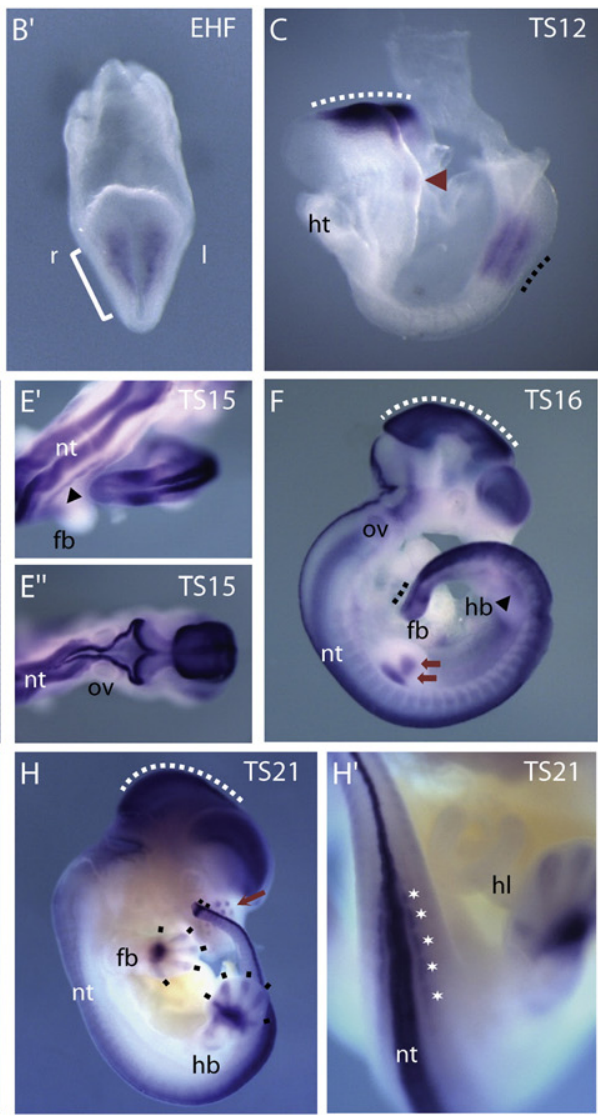
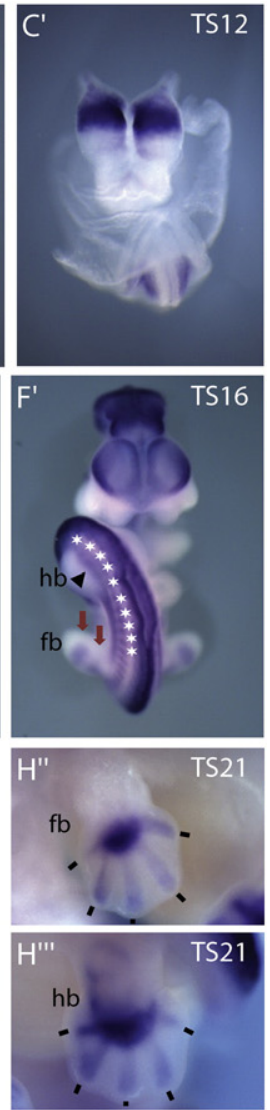

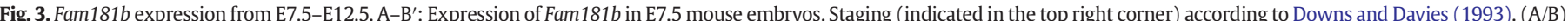

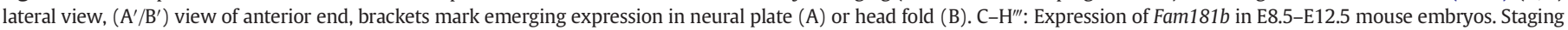

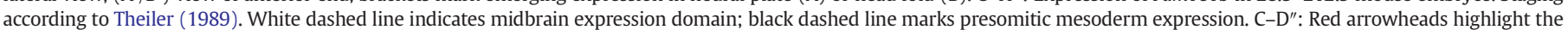

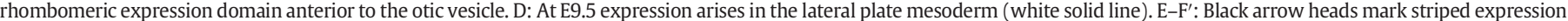

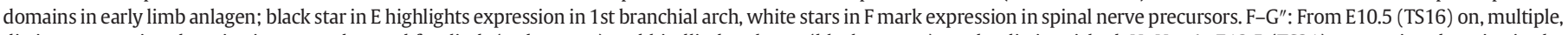

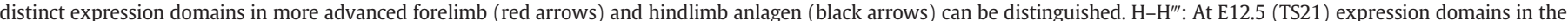

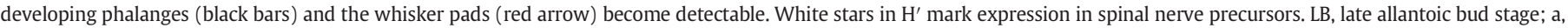
anterior; p, posterior; l, left; r, right; EHF, early head fold stage; TS, Theiler stage; ht, heart tube; nt, neural tube; ov, otic vesicle; fb, forelimb bud; hb, hindlimb bud.

transcription during somitogenesis. Additional expression domains at TS14 were detected in the telencephalon, in the closed neural tube, and the lateral plate mesoderm (lpm) (Fig. 3D, solid white line). The signal in the neural tube was mainly localized to the medial portion (Fig. 4D/D') and extended from the caudal end to the otic vesicle (ov). The signal detected in the lpm was strongest around the level of the prospective forelimb bud. At the morphological onset of formation of the forelimb bud (TS15), strong expression emerged as a single domain in the medial portion of the limb bud, while at the level of the prospective hindlimb bud an additional strong signal was detected in the lpm (Fig. 3E). At this stage, a small domain of Fam $181 b$ transcriptional activity was also detectable in the anterior portion of the first branchial arch (black star). Additionally, neural tube expression was observed in the roof plate, starting in the hindbrain and progressing posteriorly (Fig. 3E-G, Fig. $\left.4 \mathrm{~B} / \mathrm{B}^{\prime}, \mathrm{D} / \mathrm{D}^{\prime}\right)$. Notably, while the signal in the medial neural tube was absent from the hindbrain region around the ov (Fig. 3D"), the roof plate expression was continuous throughout the hindbrain and trunk (Fig. 3E", G/G"). At TS16 the emerging forelimb bud showed a second, more distal Fam181b expression domain, while the hindlimb bud began to recapitulate the expression pattern seen earlier within the forelimb bud, with a single medial domain (Fig. $3 \mathrm{~F} / \mathrm{F}^{\prime}$, red and black arrows). From the dorsal side of the neural tube we observed triangular-shaped extensions of the Fam181b signal along the trunk which extended ventrally (Fig. $3 \mathrm{~F}^{\prime}$, asterisks). We identified these as cells of the peripheral nervous system, such as those which form the dorsal root ganglia (Fig. 4D-E'). Expression was also detected in the dermomyotome (the dorsolateral compartment of differentiating somites) along the length of the trunk (Fig. 3F', $\mathrm{G}^{\prime} / \mathrm{G}^{\prime \prime \prime}$, Fig. $4 \mathrm{E} / \mathrm{E}^{\prime}$ ).

At TS18 the distal expression domain in the forelimb bud was extended (Fig. $3 \mathrm{G} / \mathrm{G}^{\prime}$ ). This was present as a distally positioned stripe and two weaker proximodistally expanded stripes. The first corresponds to the position of wrist plate progenitors, while the latter likely correspond to the chondrogenic progenitors of the ulna and radius. At this stage, the hindlimb bud also showed a second, more distal, and slightly proximodistally extended Fam 181 b-expressing area (Fig. 3G'"'). After formation of hand and foot plate at TS21, both forelimb and hindlimb anlagen exhibited signals in the forming digits (Fig. 3H, black bars). While the staining in ulnar and radial regions of the forelimb were undetectable at this stage, the anlagen of tibia and fibula in the hindlimb showed Fam $181 \mathrm{~b}$ expression (compare Fig. $3 \mathrm{H}^{\prime \prime}$ to Fig. $3 \mathrm{H}^{\prime \prime \prime}$ ). Additional staining appeared in the whisker pads at this stage (Fig. $3 \mathrm{H}$, red arrow).

To further examine the differential domains of expression in the limbs, E14.5 forelimbs and hindlimbs were dissected and subjected to WISH for Fam 181b, followed by vibratome sectioning. This revealed that the expression detectable in the outgrowing digits of E14.5 limbs (Fig. 4F/G) was mainly localized to the cartilaginous regions between the phalanges, corresponding to the prospective joints (Fig. $4 \mathrm{H}-\mathrm{K}$ ). 

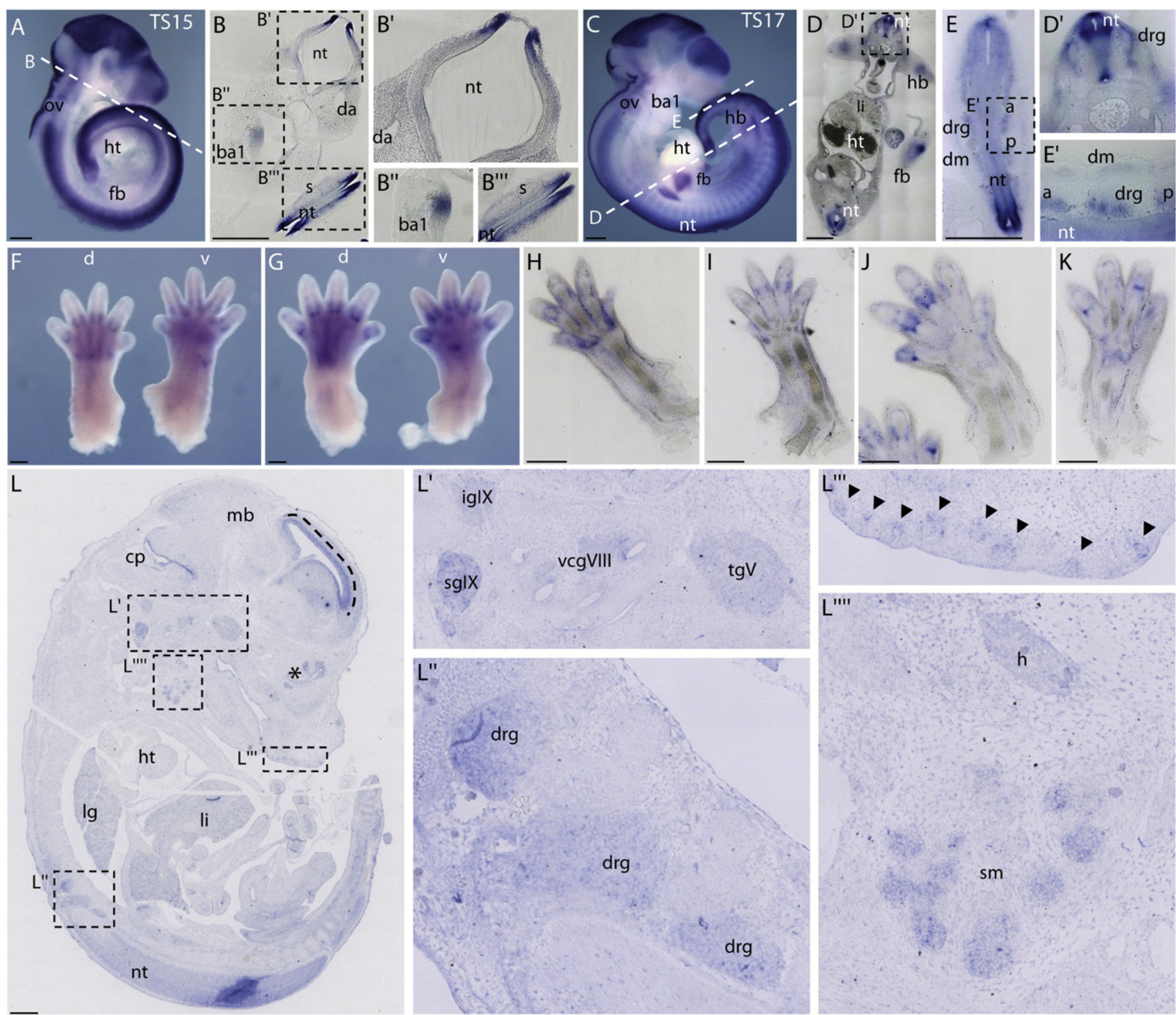

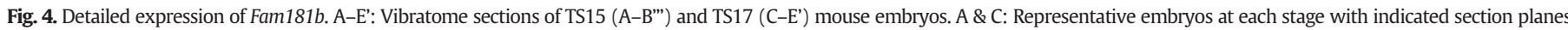

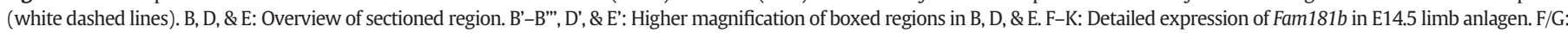

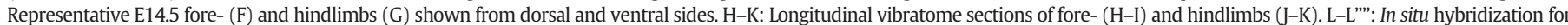

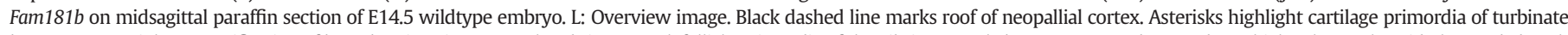

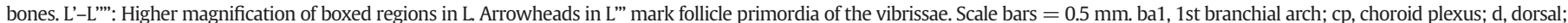

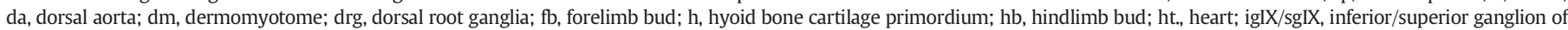

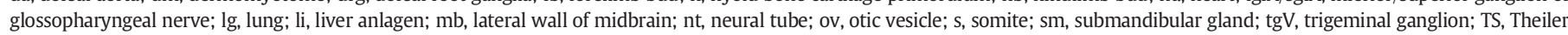
stage; v, ventral; vcgVIII, vestibulocochlear ganglion.

In situ hybridization for Fam181b on midsagittal paraffin sections from E14.5 embryos (Fig. 4L-L"'") showed a strong signal within the ventricular zone of the forebrain, midbrain and the neural tube (Fig. 4L), confirmed expression in the dorsal root ganglia (Fig. 4L"), and revealed expression in cranial ganglia and nerves V, VIII, and IX (Fig. 4L'), the placodes of the vibrissal follicles (Fig. 4L"'), and the submandibular gland (Fig. 4L"'"). Expression was detected in cartilaginous primordia of the hyoid and turbinate bones. The simultaneous presence of Fam $181 \mathrm{~b}$ mRNA in ganglia, nerves, and cartilaginous structures points to a common origin for these cells from the neural crest. This is compatible with the signals seen at earlier stages within the first branchial arch and extending from the neural tube along the trunk.

In summary, Fam181b shows a highly dynamic expression pattern during mouse development, with strong expression domains detected in various neural tissues during all stages investigated. Transient expression was also detected in the mesenchymal psm and limb anlagen, especially within its cartilage, and within neural crest derivatives.

\subsection{Fam181a is differentially expressed during development and partially overlaps with Fam181b}

Next we examined expression of the Fam181b paralog, Fam181a, during murine development by performing WISH on wt embryos between E7.5 and E12.5. Whereas Fam181b expression was detected in embryos as young as late allantoic bud stage, Fam181a expression first became detectable at the late headfold stage in the prospective midbrain region (Fig. 5A-B', bracket, cf. Fig. 3A-B'). Both genes were 

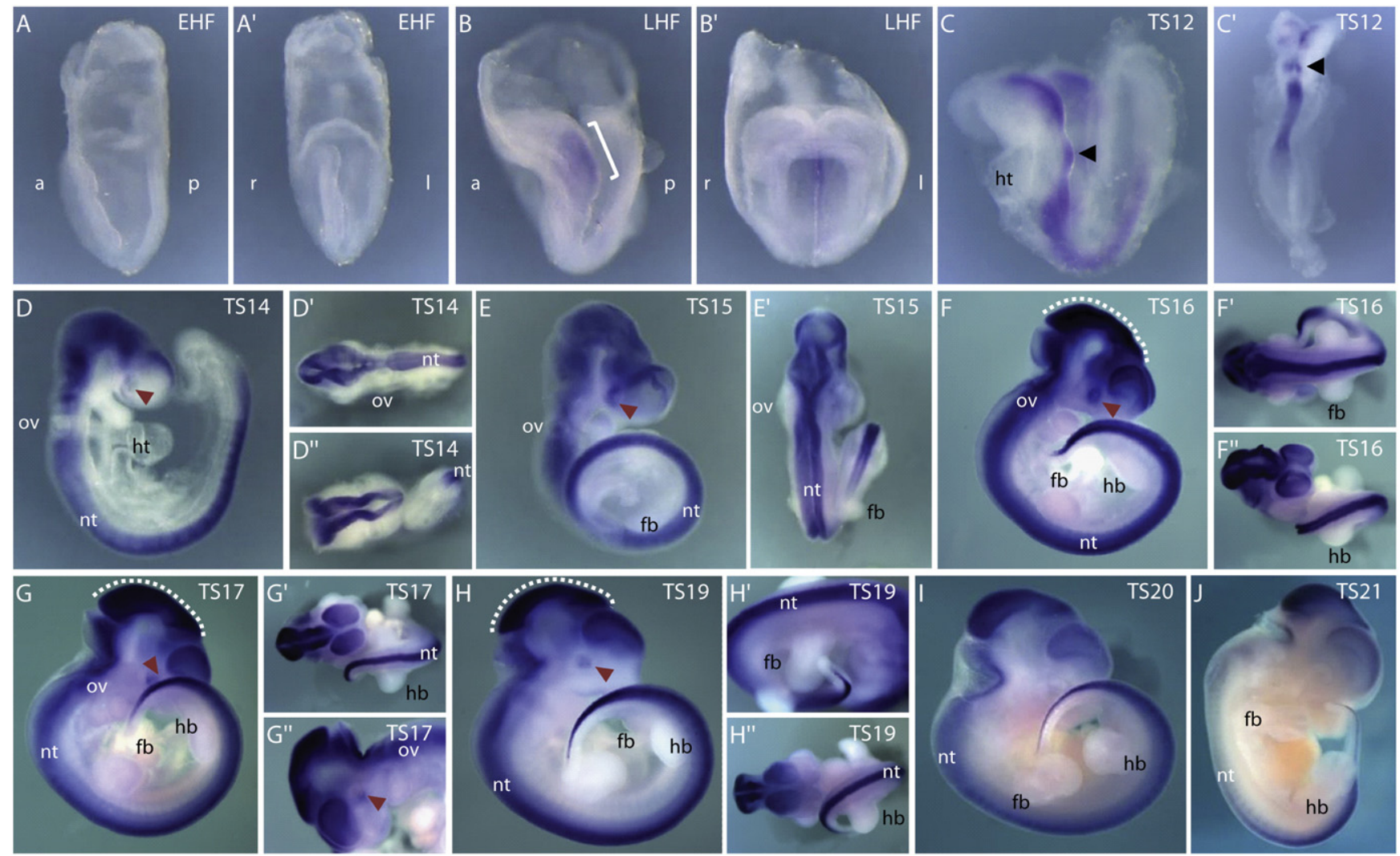

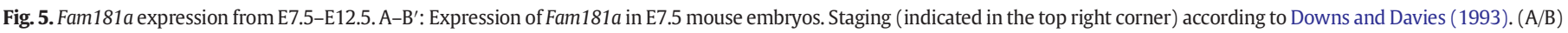

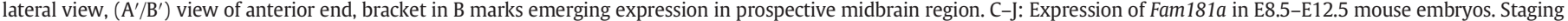

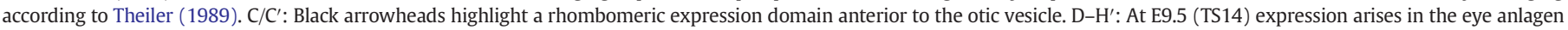

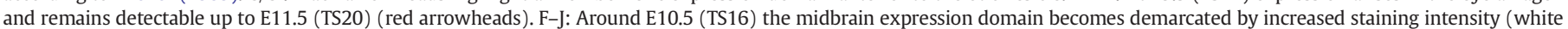

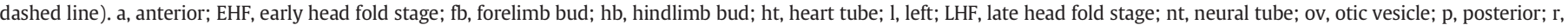
right; TS, Theiler stage.

found to be highly expressed in the developing midbrain. Fam181a expression extended throughout the entire neural tube by TS12

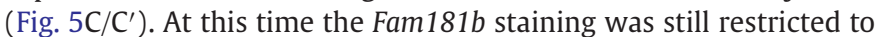
the midbrain region, and started to arise in the psm, whereas Fam181a was never detected in the psm at any stage investigated. A distinct signal for Fam181a could be detected in the rhombencephalon, anterior to the ov (Fig. $5 \mathrm{C} / \mathrm{C}^{\prime}$, black arrowhead). This domain corresponded to a similar expression domain observed for Fam181b (compare to Fig. 3C, red arrowhead). At later stages, Fam181 a transcription remained limited mainly to the neural tube and the developing brain, except for a domain in the eye anlagen (Fig. 5D-H, red arrowhead). At TS14, the Fam181a signals in the brain vesicles and in the neural tube were separated by a small gap at the level of the ov (Fig. 5D'). A similar gap was observed for Fam $181 \mathrm{~b}$ neural tube expression, which remained detectable up to TS16 (compare to Fig. 3D-F'), while for Fam181a it was only observed at TS14.

Between TS16 and TS19, the midbrain domain of Fam181a showed an increased staining intensity, demarcating it from the surrounding neural expression domains (Fig. $5 \mathrm{~F}-\mathrm{H}^{\prime \prime}$, white dashed line). This is similar to the Fam181b midbrain domain, which was distinctive from other neural expression domains at all stages investigated (compare to Fig. 3D-H). Transcription of both paralogs differed within the developing limb buds. While Fam181b showed continuous, distinctive limb expression starting at TS15 (see Figs. 3E-H"', 4C-K), no signal could be detected for Fam181a in the limbs up to TS21 (Fig. 5E-J).

Overall, Fam181a transcriptional activity appears mainly to be limited to neural structures, where it shows extensive overlap with Fam 181 b. In line with these findings, the expression of both genes was found to increase during the differentiation of murine ESCs into the neural lineage according to Bibel et al. (2007) (Fig. 6A). During the differentiation procedure, the highest expression levels for both genes were observed during differentiation into neural progenitor (NP) cells, while they decreased again during terminal differentiation into neurons. The overlapping expression in neural tissues might be indicative of functional redundancy for the paralogs in these tissues. In fact, our investigations revealed lack of any obvious morphological phenotype in mice which were homozygous-null for Fam181b alone (Fig. 9).

\subsection{Fam181 $a$ and $b$ are expressed in various tissues of the adult mouse}

In order to examine expression of both Fam181 genes in adult tissues, we performed RT-PCR and real-time qPCR on cDNA from various selected organs isolated from a female mouse (Fig. 6B).

The highest expression levels were found in the cerebrum and cerebellum, demonstrating maintenance of the neural expression for both genes after embryonic development. Together with the data obtained from the embryos and the in vitro differentiation, this strongly suggests a main role for the Fam181 gene family in neural tissues. This notion is further supported by findings from in vitro mouse models for the neurodevelopmental disorders Pitt-Hopkins syndrome and 9q34 deletion syndrome having dysregulated Fam181a expression (Chen et al., 2014). The decreasing expression levels during the terminal differentiation of neural progenitors into neurons might be indicative of a function in non-neuronal cell types of the nervous system. In line with this, Fam $181 \mathrm{~b}$ transcripts 

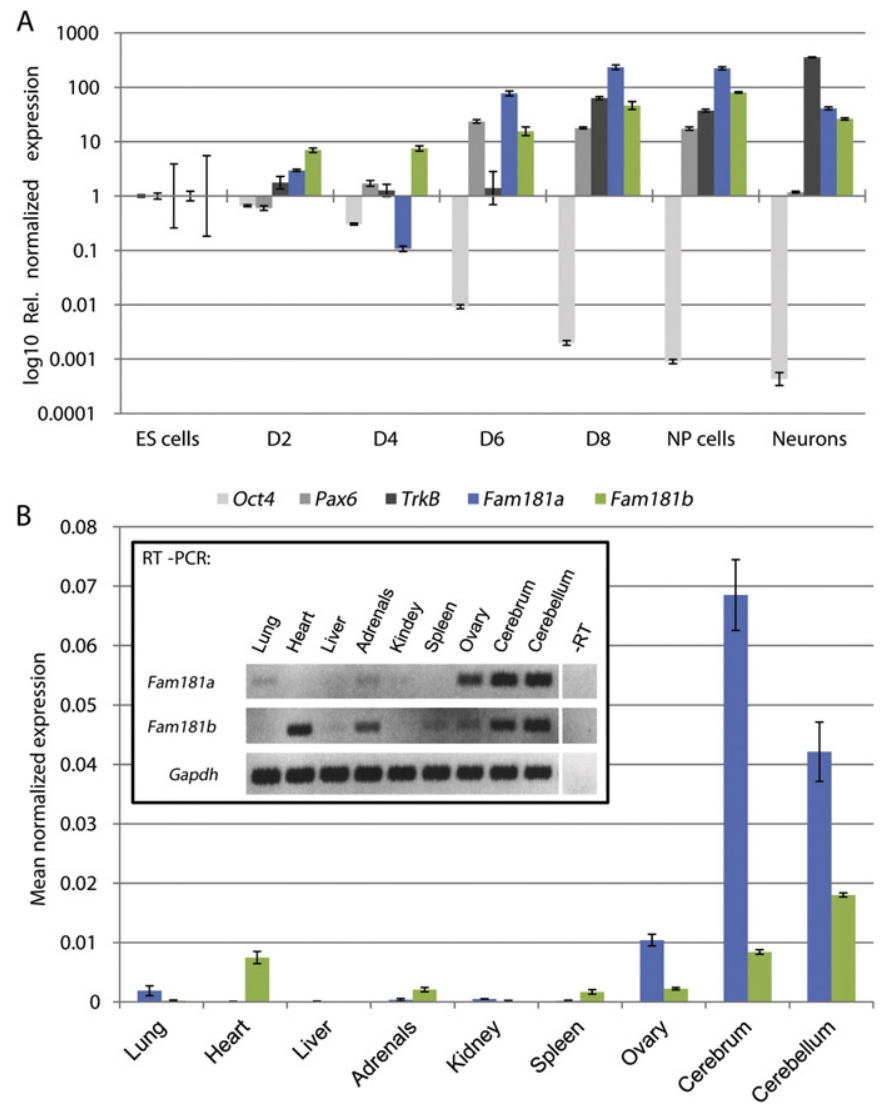

Fig. 6. Fam181 expression in ES cells differentiated into the neural lineage and in adult tissues. A: Neural differentiation of murine F1G4 ES cells according to Bibel et al. (2007). During the differentiation procedure samples were taken following formation of the cellular aggregates and used for total RNA extraction and cDNA synthesis. Fam181a/b expression levels were analyzed by quantitative real-time PCR. Oct4 was used as a stem cell marker Pax6 as a marker for neural differentiation, and TrkB for terminal neurons. The expression of Fam181a is maximally upregulated in the D8 sample (230-fold), that of Fam181b in the neural progenitor (NP) cell sample (80-fold). B: Fam181 $a$ and $b$ expression in various tissues of an adult female mouse. Total RNA was extracted from selected organs and reversetranscribed into cDNA. A sample treated without reverse transcriptase was used as negative control. The cDNA was either used for RT-PCR with Gapdh as loading control (inset), or for quantitative real-time PCR (graph). qPCR was normalized to Pmm2 and analyzed by qGene.

have been found to be enriched in the transcriptome of astrocytes (Lovatt et al., 2012). Further overlapping expression was found in the ovaries and adrenals (Fam181a only weakly), though at lower levels as compared to the neural expression.

Unique expression for Fam181a was observed in the lung. Changes in the CpG-methylation of the Fam181a locus have been reported to be associated with asthma (Gunawardhana et al., 2014; Wysocki et al., 2014), and breathing difficulties is also one of the symptoms of PittHopkins syndrome. This may suggest a link between the dysregulation of Fam181a expression and lung function. Unique expression for Fam181b was found in the heart and spleen. Very faint bands from the RT-PCR were also detected in liver (Fam181a and $b$ ) and kidney (Fam181a), though these were barely detectable by real-time qPCR. In general, the expression levels for both Fam181 genes in all tissues investigated were lower than those of the housekeeping gene Pmm2, which was used for normalization.

\subsection{Fam181b oscillates in the psm in-phase with Notch targets}

Fam $181 \mathrm{~b}$ was previously predicted to exhibit oscillatory expression during somitogenesis (Dequéant et al., 2006). First we checked whether this oscillation could be visualized by WISH using E9.5 mouse embryos. Comparing different embryos we found changes in the anteroposterior extension of Fam $181 \mathrm{~b}$ mRNA within the psm, varying from a broad domain extending throughout the posterior psm, to a narrow domain of expression at the level of the prospective somite S-II (Fig. 7A-A", white bracket). Expression in the caudal end/tail bud was never found to be stronger than in more anterior regions of the PSM, as it is the case for other oscillating genes like Lfng (Forsberg et al., 1998) and Dkk1 (Dequéant et al., 2006). To verify that the observed changes were due to oscillating expression, we went on to perform tail-half cultures, wherein the caudal trunk of TS13-15 embryos was split at the midline and one half was fixed $(t=0)$, while the second half was further cultured for $90 \mathrm{~min}$ or $120 \mathrm{~min}$ before fixation. Both halves were then simultaneously subjected to in situ-hybridization for Fam181b. After 90 min, 8 out of 8 samples showed changes in Fam181b mRNA distribution (Fig. 7B). In contrast, after $120 \mathrm{~min}$, the time for one complete somitogenic cycle in the mouse, a comparable pattern was observed between the cultured halves and their counterparts (Fig. $7 \mathrm{~B}^{\prime}, \mathrm{n}=4$ ). This verifies that Fam $181 \mathrm{~b}$ is indeed expressed in an oscillatory manner during somitogenesis.

Next, to address which signaling pathway the Fam $181 \mathrm{~b}$ oscillations were associated with, we prepared tail halves from E9.5 mouse caudal ends and subjected the two halves to in situ-hybridization for Fam181b, and either Dkk1 (Fig. 7C/C') or Lfng (Fig. 7D/D'). During the phase when the transcriptional domain of the Wnt-target Dkk1 (Niida et al., 2004; Dequéant et al., 2006) was expanded throughout the posterior $2 / 3$ of the psm, Fam $181 \mathrm{~b}$ mRNA was restricted to a small stripe at the level of the prospective somite S-II (Fig. 7C). When Dkk1 expression was turned off, the Fam181b signal was expanded through the psm (Fig. 7C'). In contrast, Fam $181 \mathrm{~b}$ expression could be detected around the level of S-II when the mRNA of the Notch target gene Lfng (see Forsberg et al., 1998; McGrew et al., 1998; Aulehla and Johnson, 1999; Morales et al., 2002) was restricted to the S-I prospective somite region (Fig. 7D). When Lfing was strongly expressed in the caudal end and posterior psm (with a stripe in the anterior half of the S0 somite), the Fam 181 b domain extended more posteriorly (Fig. 7D'). This demonstrates that oscillation of Fam $181 \mathrm{~b}$ is in-phase with the Notch-target gene Lfng and out-of-phase with the canonical Wnt-target Dkk1. In the anterior psm, Lfng expression becomes stabilized as a stripe of expression through the activity of the transcription factor MESP2, thereby inhibiting Notch signaling in the So region (Morimoto et al., 2005; Oginuma et al., 2010). Interestingly, Fam $181 \mathrm{~b}$ is absent from this region (red line in Fig. 7D'), suggesting a direct regulation by Notch signaling.

To investigate whether Notch signaling activity has a regulatory impact on Fam $181 \mathrm{~b}$ transcription, we performed WISH on loss-of-function mutants for Dll1 (Hrabe de Angelis et al., 1997) and their heterozygous and wild-type control littermates. Dll1, a ligand for the Notch receptor, is expressed in the psm and in the central nervous system, with a strong domain in the developing forebrain (Tax et al., 1994; Bettenhausen and Gossler, 1995; Bettenhausen et al., 1995). Homozygous mutants can be discriminated from their wild-type and heterozygous counterparts by defects in segmental patterning, which become overt from E8.5 onwards (Hrabe de Angelis et al., 1997). In E9.5 wild-type and heterozygous control embryos, Fam181b expression was present as described above (Fig. 7E, compare to Fig. 3D). In contrast, Fam181b transcripts were absent from the psm of Dll1 ${ }^{-1-}$ embryos (Fig. 7E') and expression in the telencephalon was restricted to its dorsal aspect (black arrowhead in Fig. 7E', compare to Fig. 7E). Taken together, this suggests a regional dependency of Fam181b expression on Notch signaling, especially in the psm and the telencephalon. It has been previously shown that cyclic activity of the Notch pathway leads to a salt-and-pepperlike oscillatory expression of Dll1 and Hes 1 in neural progenitor cells (Kageyama et al., 2008; Shimojo et al., 2008). It remains to be investigated whether Fam $181 \mathrm{~b}$ activity also displays oscillatory expression in this cell type. 

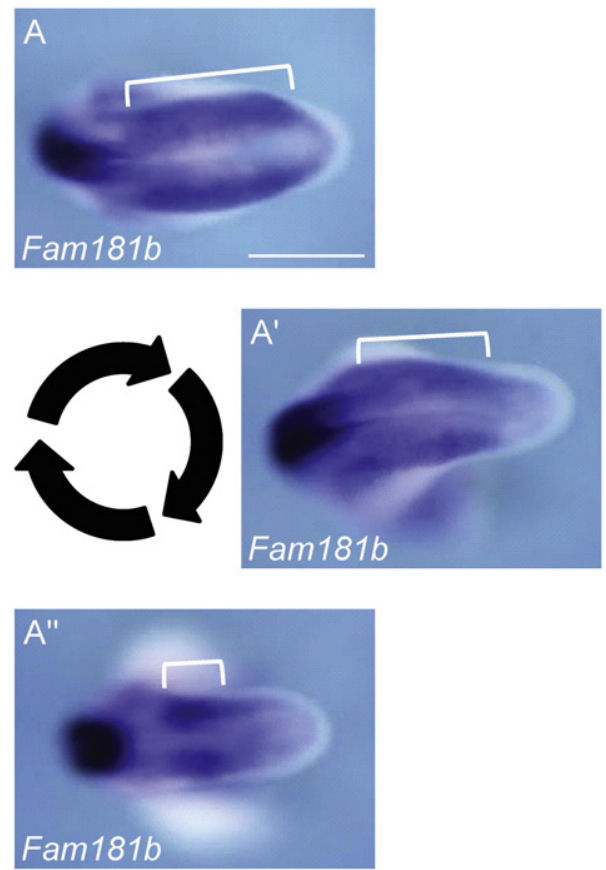
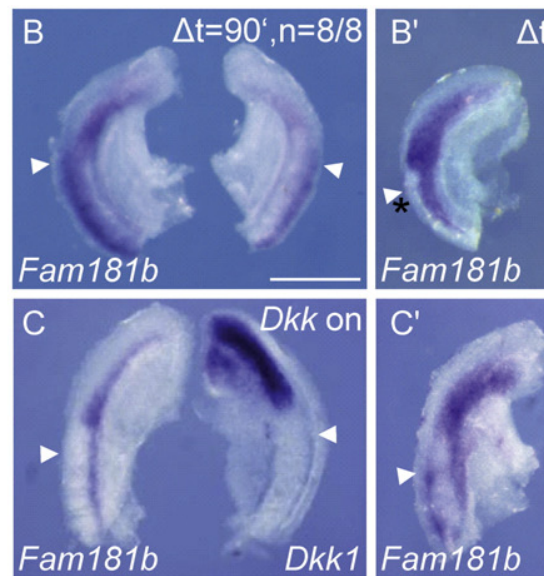

$\Delta t=120^{\prime}, n=4 / 4$
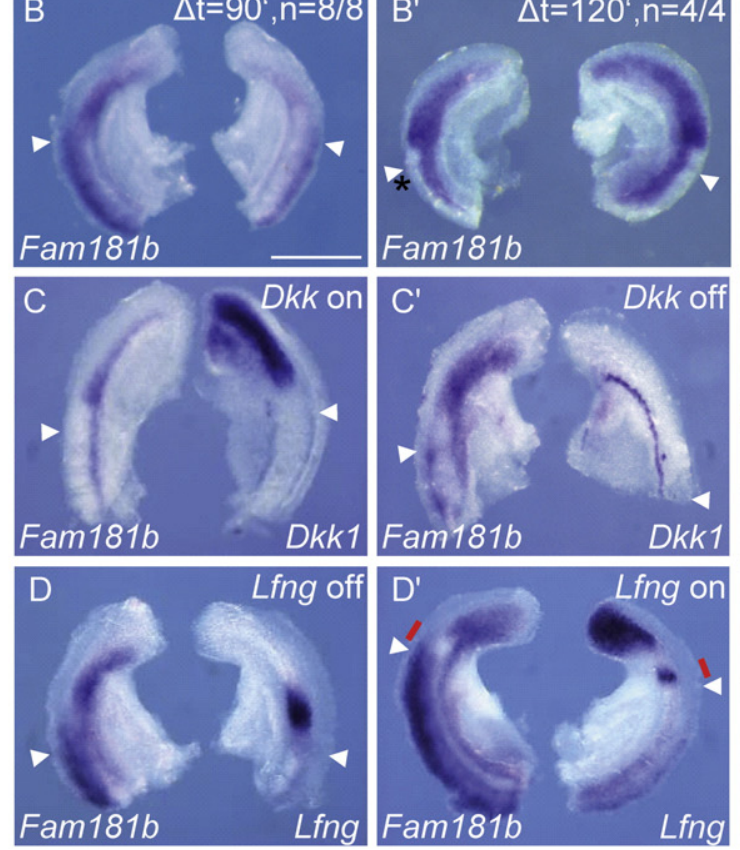
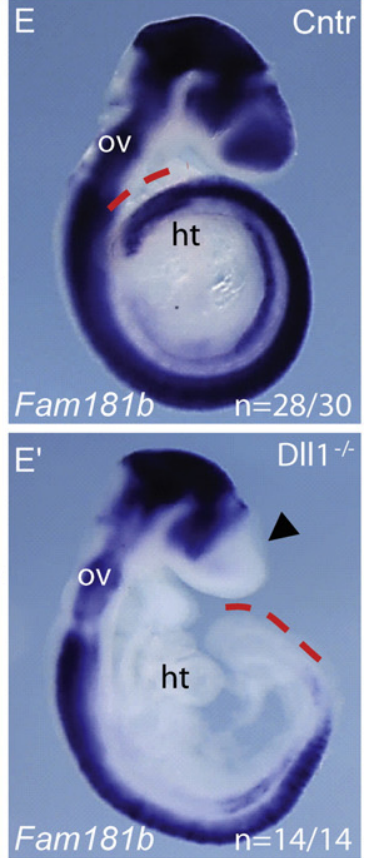

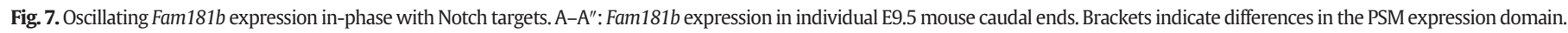

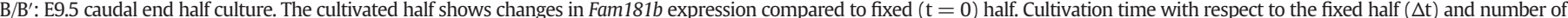

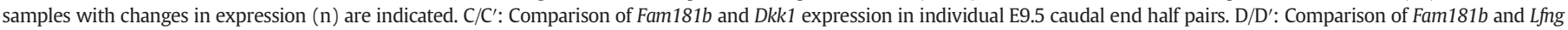

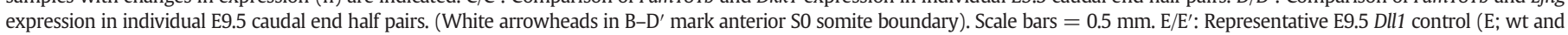
het) and Dll1 ${ }^{-1-}\left(E^{\prime}\right)$ embryos from their ventral sides. Red dashed line highlights the psm. Numbers of embryos are indicated in the bottom right corner. ht, heart; ov, otic vesicle.

\subsection{The psm and lpm expression of Fam $181 \mathrm{~b}$ is dependent on genetic background}

To gain further insight into the regulation of Fam181b transcription, we analyzed the Fam181b expression pattern in Dkk1 loss-of-function embryos (Mukhopadhyay et al., 2001). While most expression domains appeared unaltered, presomitic and lateral plate mesoderm expression of Fam $181 \mathrm{~b}$ was undetected in all of the embryos investigated, irrespective of their genotype (data not shown). Our Fam181b expression analysis was performed in embryos from CD1 and NMRI outbred strains, and the Dll1 mouse line was maintained on a CD1 background, whereas the Dkk1 mutation is maintained on $129 \mathrm{~S} 2 \mathrm{SvHsd}$ or C57BL/6J backgrounds. Assimilating this information, we presumed that the genetic background may be impacting the Fam $181 \mathrm{~b}$ expression pattern. To investigate this notion further, we analyzed E10.5 embryos from C57BL/ $6 \mathrm{~J}$ and $129 S 2 S v H s d$ inbred strains by WISH. In contrast to CD1 embryos, which were processed and stained in parallel (Fig. 8A/ $\mathrm{A}^{\prime}$ ), neither C57BL/6 J (Fig. 8B/B' ${ }^{\prime}$ nor 129S2SvHsd embryos (Fig. 8C/C') exhibited expression of Fam $181 \mathrm{~b}$ in the psm or lpm. All other domains of Fam $181 \mathrm{~b}$ expression described above, including those in the mesodermal-derived limb anlagen, were maintained (see Fig. 3 ) and were of comparable staining intensity with respect to CD1 controls. This argues for a partial dependency of mesodermal Fam181b expression on genetic background. In support of our results, such backgroundspecific differences for Fam181b between mouse strains were also identified in a transcriptome analysis by Kong et al. (2014). These results underpin that the use of a particular genetic background is an important consideration for the comparability of experiments in the mouse, especially, although not exclusively, with respect to the interpretation and validation of expression data. For instance, the screens that identified Fam $181 \mathrm{~b}$ as an oscillatory gene were done using either the CD1 (Dequéant et al., 2006) or the NMRI outbred strain (P. Grote, L. Wittler, M. Werber, and B.G. Herrmann, unpublished data). In contrast, functional analyses are predominantly performed using inbred strains, such as C57BL/6. Although the exact genetic mechanism of this tissue-specific background-dependency remains to be investigated, to our knowledge this is the first description of a cycling gene exhibiting backgrounddependent oscillatory expression.

\subsection{Loss of Fam181b does not result in an overt morphological phenotype}

To investigate the function of the Fam $181 \mathrm{~b}$ gene, we generated a conditional allele by homologous recombination in murine ESCs, which was then used to generate a loss-of-function model (Fig. 9A-B). Embryos homozygous for deletion of Fam181b displayed no obvious developmental defects when examined up to E17.5 (Fig. 9C-D'). Homozygous-null offspring were vital, fertile, and displayed normal morphology (Fig. 9E). Interestingly, Fam181a expression levels were significantly reduced in brain and neural tube tissues of Fam $181 \mathrm{~b}$ null embryos compared to heterozygous littermates (Fig. 9F). Additionally, there were no changes in the expression of the neural marker genes TrkB and Pax6 in the adult cerebellum, where strong Fam181b expression is observed, and expression of the Notch target gene Lfng was similarly unaffected in heterozygous and homozygous Fam181b knock-out animals (Fig. 9G). In this adult tissue, a significant decrease in Fam181a expression levels was also detected similarly to that observed in the embryonic tissues (Fig. 9G, compare to Fig. 9F). These findings suggest that FAM181B positively regulates its paralog in these tissues. It remains to be investigated how this observation extends to other tissues. Given their similarity and partially overlapping mRNA expression patterns in developing and adult neural structures, we cannot exclude that the FAM181 proteins exhibit functionally redundant roles in the nervous system, and may also play redundant roles in a developmental context. Considering our previous data, we also cannot completely rule out the possibility of backgrounddependent phenotypic differences within the psm or lpm. 

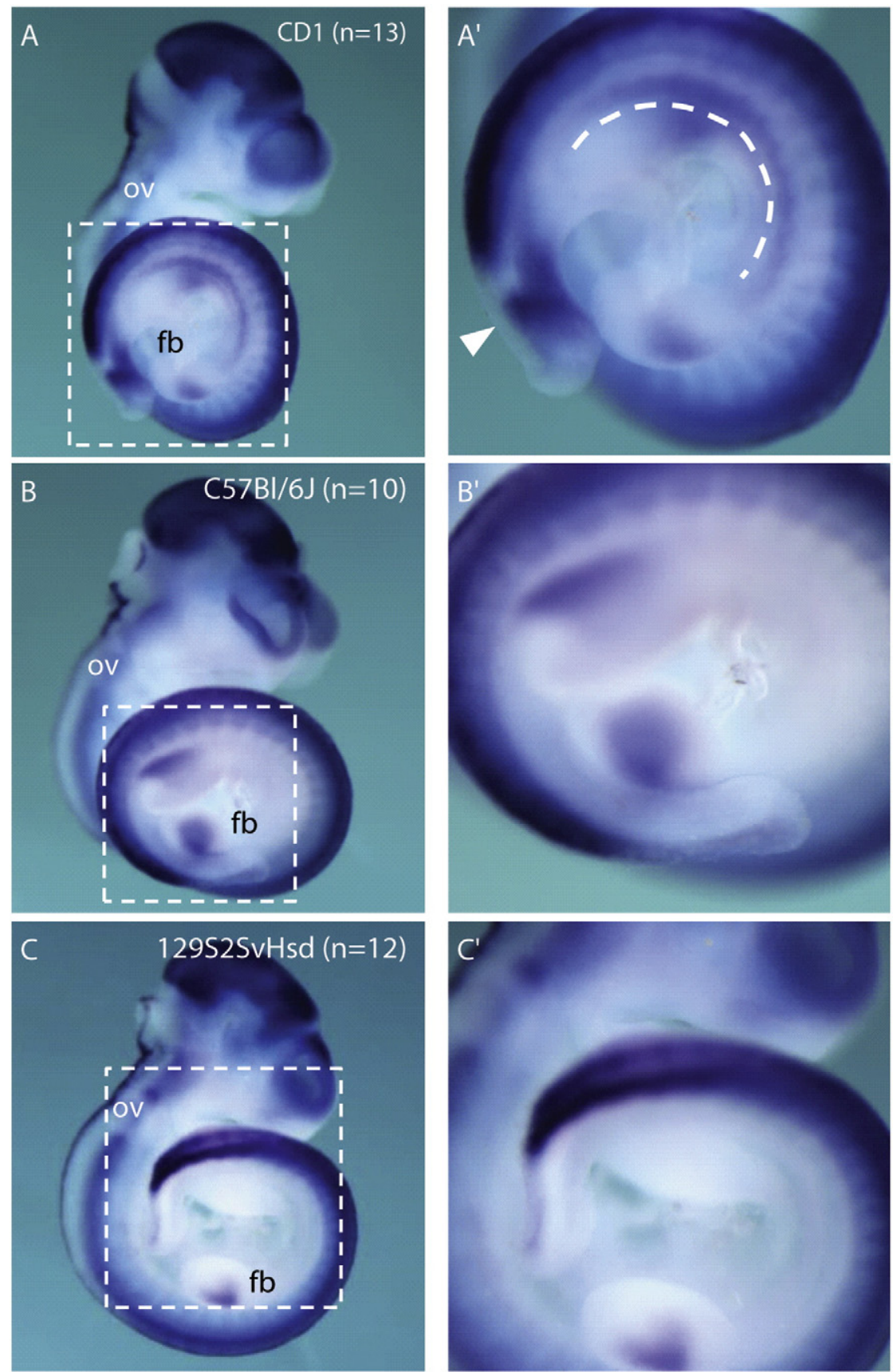

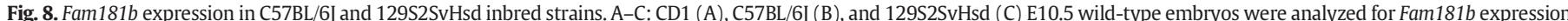

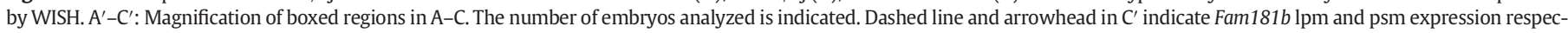
tively. fb, forelimb bud; ov, otic vesicle.

\section{Conclusions}

In summary, we have shown that the Fam181 genes constitute a novel gene family that is conserved among vertebrates with two paralogs, namely Fam181a and Fam181b, per species. Both genes display highly dynamic and specific expression patterns during murine embryonic development. Their expression is most prominent in neural tissues, where Fam181a is exclusively expressed during

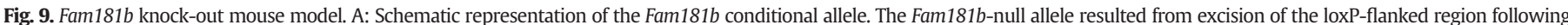

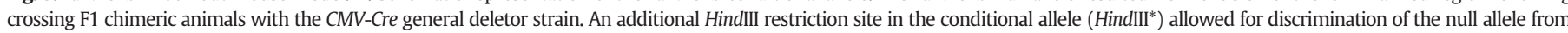

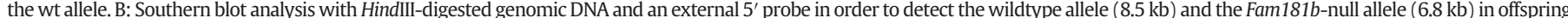

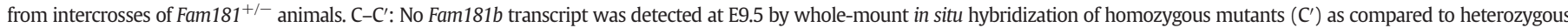

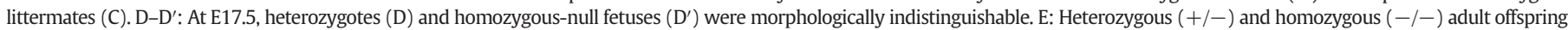

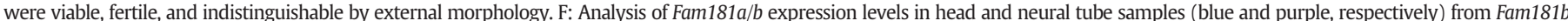

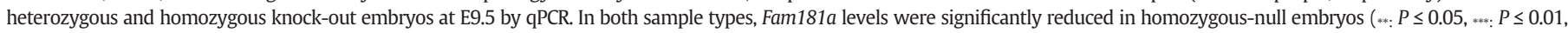

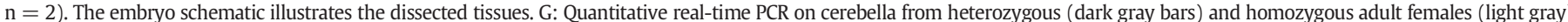

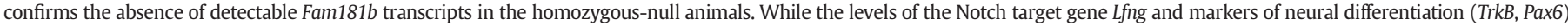

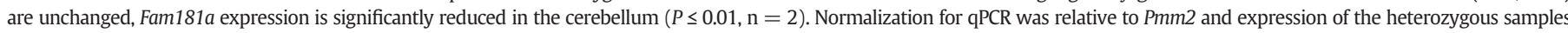
set to 1 . 


\section{A Fam181b conditionally targeted region:}

HindIII HindIII*

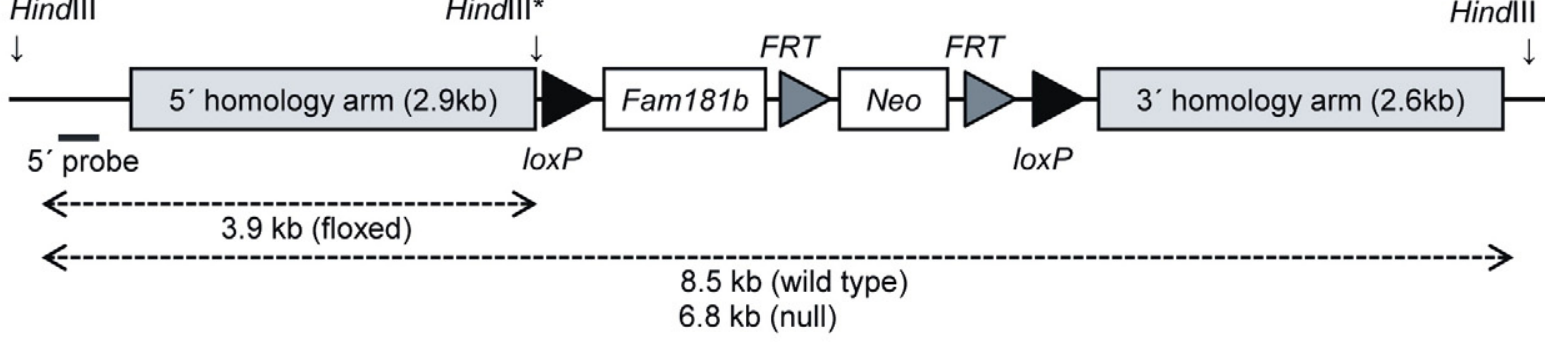

B

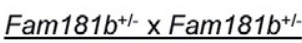

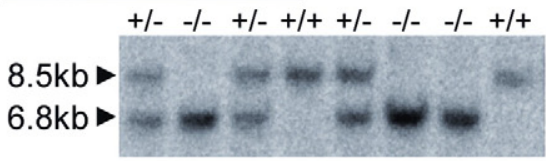

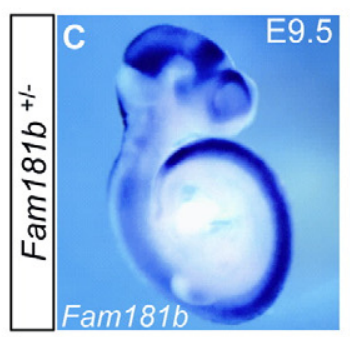
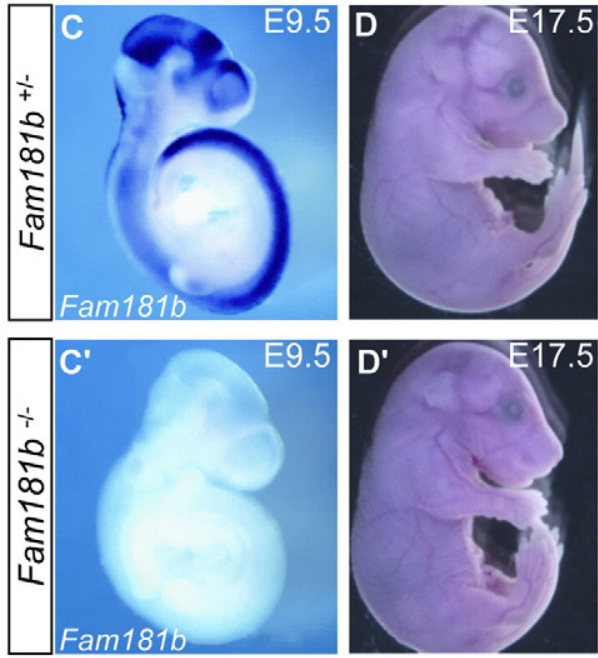

E

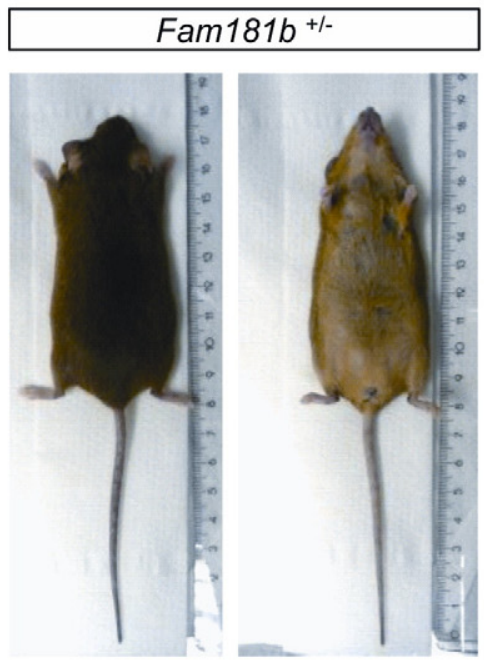

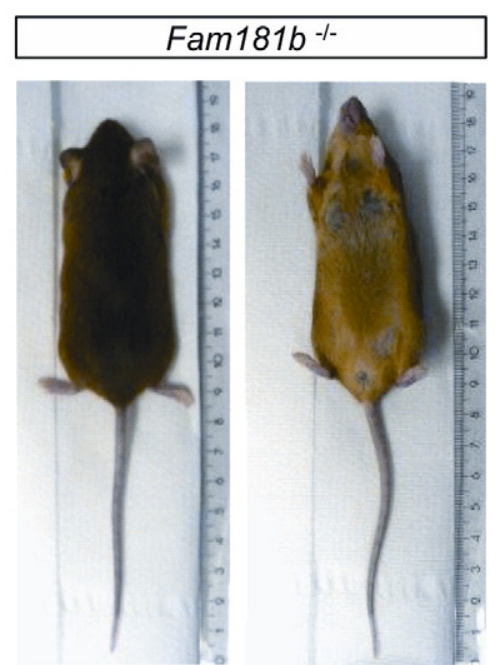

$\mathbf{F}$
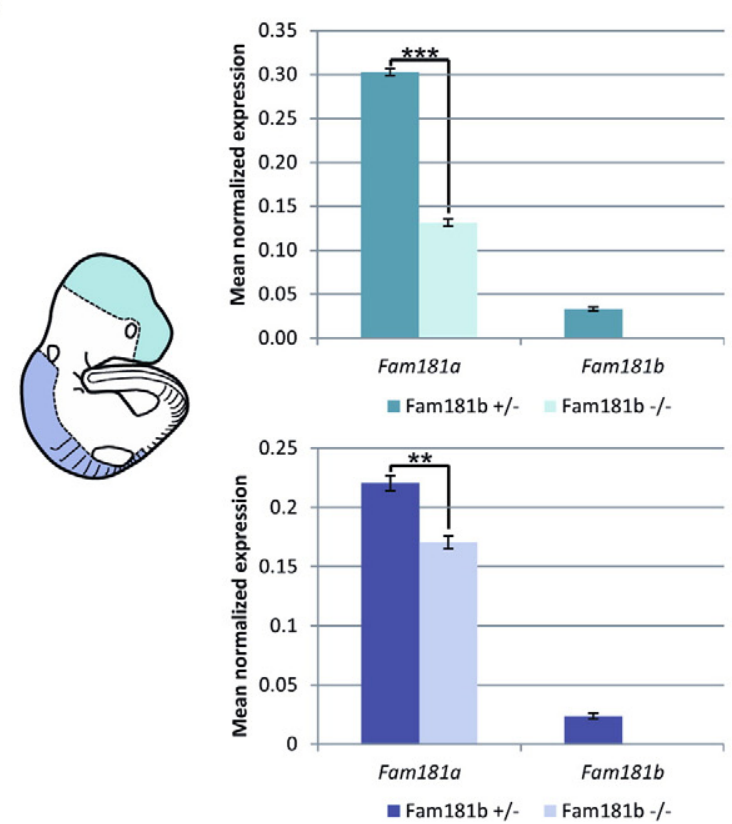

G

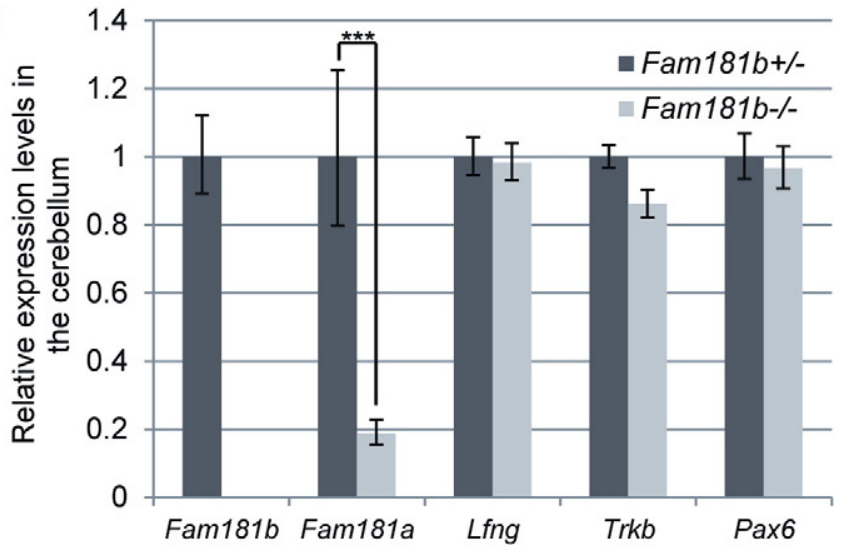


embryonic development, while Fam181b shows additional areas of transcriptional activity in mesoderm-derived tissues. We confirmed the oscillation of Fam $181 \mathrm{~b}$ transcription in mouse psm during somitogenesis, cycling in-phase with, and regulated by, the NotchDll pathway. Interestingly, the oscillating Fam $181 \mathrm{~b}$ psm expression, along with lpm expression, was found to be dependent on genetic background. The FAM181 proteins localize to the nucleus, though the responsible signal and mechanism remains to be identified. Despite its specific and diverse expression pattern, loss of Fam $181 \mathrm{~b}$ does not produce any obvious morphological phenotype in a loss-of-function mouse model, possibly due to functional redundancy with Fam181a and/or genetic background effects. Further studies are required to elucidate the functions of these proteins.

\section{Acknowledgments}

We thank Dr. Heinrich Schrewe for discussions regarding this work, Manuela Scholze and Eun-ha Shin for providing reagents, Dijana Wrembel and Christin Franke for animal husbandry, and Karol Macura and Judith Fiedler for generating mouse lines and transgenic embryos.

\section{References}

Aulehla, A., Johnson, R.L., 1999. Dynamic expression of lunatic fringe suggests a link between notch signaling and an autonomous cellular oscillator driving somite segmentation. Dev. Biol. 207 (1), 49-61 (Available at: http://www.sciencedirect.com/science/article/pii/ S0012160698991643 [Accessed September 12, 2014]).

Aulehla, A., et al., 2003. Wnt3a plays a major role in the segmentation clock controlling somitogenesis. Dev. Cell 4 (3), 395-406 (Available at: http://www.cell.com/ developmental-cell/fulltext/S1534-5807(03)00055-8 [Accessed January 19, 2014]).

Bettenhausen, B., Gossler, A., 1995. Efficient isolation of novel mouse genes differentially expressed in early postimplantation embryos. Genomics 28 (3), 436-441 (Available at: http://www.sciencedirect.com/science/article/pii/S088875438571172X [Accessed October 2, 2014]).

Bettenhausen, B., et al., 1995. Transient and restricted expression during mouse embryogenesis of Dll1, a murine gene closely related to Drosophila Delta. Development 121 (8), 2407-2418 (Available at: http://www.ncbi.nlm.nih.gov/pubmed/7671806 [Accessed October 2, 2014])

Bibel, M., et al., 2007. Generation of a defined and uniform population of CNS progenitors and neurons from mouse embryonic stem cells. Nat. Protoc. 2 (5), 1034-1043 Available at: http://dx.doi.org/10.1038/nprot.2007.147 [Accessed July 15, 2014].

Cao, X., Pfaff, S.L., Gage, F.H., 2008. YAP regulates neural progenitor cell number via the TEA domain transcription factor. Genes Dev. 22 (23), 3320-3334 (Available at: http://genesdev.cshlp.org/content/22/23/3320.short [Accessed May 7, 2014]).

Chen, L., et al., 2010. Structural basis of YAP recognition by TEAD4 in the hippo pathway. Genes Dev. 24 (3), 290-300 (Available at: http://genesdev.cshlp.org/content/24/3/ 290.short [Accessed May 2, 2014]).

Chen, E.S., et al., 2014. Molecular convergence of neurodevelopmental disorders. Am. J. Hum. Genet. (Available at: http://www.sciencedirect.com/science/article/pii/ S0002929714003966 [Accessed October 14, 2014]).

Chotteau-Lelièvre, A., Dollé, P., Gofflot, F., 2006. Expression analysis of murine genes using in situ hybridization with radioactive and nonradioactively labeled RNA probes. Methods Mol. Biol. 326, 61-87 (Available at: http://www.ncbi.nlm.nih.gov/ pubmed/16780194 [Accessed May 12, 2014]).

Cooke, J., Zeeman, E.C., 1976. A clock and wavefront model for control of the number of repeated structures during animal morphogenesis. J. Theor. Biol. 58 (2), 455-476 (Available at: http://www.sciencedirect.com/science/article/pii/S0022519376801312 [Accessed March 23, 2014]).

Del Corral, R.D., et al., 2003. Opposing FGF and retinoid pathways control ventral neural pattern, neuronal differentiation, and segmentation during body axis extension. Neuron 40 (1), 65-79 (Available at: http://www.sciencedirect.com/ science/article/pii/S0896627303005658 [Accessed March 23, 2014]).

Dequéant, M.-L., et al., 2006. A complex oscillating network of signaling genes underlies the mouse segmentation clock. Science (New York, N.Y.) 314 (5805), 1595-1598 (Available at: http://www.sciencemag.org/content/314/5805/1595.short [Accessed March 23, 2014]).

Downs, K., Davies, T., 1993. Staging of gastrulating mouse embryos by morphological landmarks in the dissecting microscope. Development 118 (4), 1255-1266 (Available at: http://dev.biologists.org/content/118/4/1255.short [Accessed July 16, 2014]).

Dubrulle, J., McGrew, M.J., Pourquié, O., 2001. FGF signaling controls somite boundary position and regulates segmentation clock control of spatiotemporal Hox gene activation. Cell 106 (2), 219-232 (Available at: http://www.cell.com/fulltext/S00928674(01)00437-8 [Accessed March 23, 2014]).

Eakin, G.S., Hadjantonakis, A.-K., 2006. Production of chimeras by aggregation of embryonic stem cells with diploid or tetraploid mouse embryos. Nat. Protoc. 1 (3), 1145-1153 (Available at: http://www.nature.com/nprot/journal/v1/n3/pdf/nprot.2006.173.pdf [Accessed May 12, 2014]).
Forsberg, H., Crozet, F., Brown, N.A., 1998. Waves of mouse Lunatic fringe expression, in four-hour cycles at two-hour intervals, precede somite boundary formation. Curr. Biol. 8 (18), 1027-1030 (Available at: http://www.sciencedirect.com/science/ article/pii/S0960982207004241 [Accessed September 12, 2014]).

Gunawardhana, L.P., et al., 2014. Differential DNA methylation profiles of infants exposed to maternal asthma during pregnancy. Pediatr. Pulmonol. 49 (9), 852-862 (Available at: http://www.ncbi.nlm.nih.gov/pubmed/24166889 [Accessed November 1, 2014]).

Hrabe de Angelis, M., McIntyre, J., Gossler, A., 1997. Maintenance of somite borders in mice requires the Delta homologue DII1. Nature 386 (6626), 717-721 (Available at: http://dx.doi.org/10.1038/386717a0 [Accessed October 2, 2014]).

Kageyama, R., et al., 2008. Dynamic Notch signaling in neural progenitor cells and a revised view of lateral inhibition. Nat. Neurosci. 11 (11), 1247-1251 (Available at: http://dx.doi.org/10.1038/nn.2208 [Accessed September 16, 2014]).

Kelley, L.A., Sternberg, M.J.E., 2009. Protein structure prediction on the Web: a case study using the Phyre server. Nat. Protoc. 4 (3), 363-371 (Available at: http://dx.doi.org/10. 1038/nprot.2009.2 [Accessed April 29, 2014]).

Kong, S.W., et al., 2014. Divergent dysregulation of gene expression in murine models of fragile X syndrome and tuberous sclerosis. Mol. Autism 5 (1), 16 (Available at: http://www.pubmedcentral.nih.gov/articlerender.fcgi?artid=3940253\&tool= pmcentrez\&rendertype $=$ abstract $[$ Accessed October 29, 2014]).

Larkin, M.A., et al., 2007. Clustal W and Clustal X version 2.0. Bioinformatics 23 (21), 2947-2948 (Available at: http://bioinformatics.oxfordjournals.org/content/23/21 2947.short [Accessed March 19, 2014]).

Liu, P., Jenkins, N.A., Copeland, N.G., 2003. A highly efficient recombineering-based method for generating conditional knockout mutations. Genome Res. 13 (3), 476-484 (Available at: http://genome.cshlp.org/content/13/3/476.short [Accessed April 28, 2014]).

Lovatt, D., et al., 2012. The astrocyte transcriptome. Neuroglia (Available at: http:// books.google.de/books?hl=de\&lr=\&id=XPI6jCVk7BsC\&oi=fnd\&pg=PA347\& $\mathrm{dq}=$ fam181b\&ots $=42 \mathrm{dPvpFGDm} \& \mathrm{sig}=$ yOeled8NQ2ZOS8zzTwIFXGLc7f4 [Accessed October 29, 2014]).

McGrew, M.J., et al., 1998. The lunatic fringe gene is a target of the molecular clock linked to somite segmentation in avian embryos. Curr. Biol. 8 (17), 979-982 (Available at: http://www.sciencedirect.com/science/article/pii/S0960982298704014 [Accessed September 12, 2014]).

Morales, A.V., Yasuda, Y., Ish-Horowicz, D., 2002. Periodic lunatic fringe expression is controlled during segmentation by a cyclic transcriptional enhancer responsive to Notch signaling. Dev. Cell 3 (1), 63-74 (Available at: http://www.sciencedirect.com/ science/article/pii/S1534580702002113 [Accessed September 12, 2014]).

Moreno, T.A., Kintner, C., 2004. Regulation of segmental patterning by retinoic acid signaling during Xenopus somitogenesis. Dev. Cell 6 (2), 205-218 (Available at: http:// www.sciencedirect.com/science/article/pii/S1534580704000267 [Accessed March $23,2014])$.

Morimoto, M., et al., 2005. The Mesp2 transcription factor establishes segmental borders by suppressing Notch activity. Nature 435 (7040), 354-359 (Available at: http://dx doi.org/10.1038/nature03591 [Accessed April 7, 2015]).

Mukhopadhyay, M., et al., 2001. Dickkopf1 is required for embryonic head induction and limb morphogenesis in the mouse. Dev. Cell 1 (3), 423-434 (Available at: http://www. sciencedirect.com/science/article/pii/S1534580701000417 [Accessed November 16, 2014]).

Muller, P., et al., 2002. Short technical report processing of gene expression data generated by quantitative real-time RT-PCR. Biotechniques (Available at: http://www.qpcrupdate.org/ muller-2002-qgene.pdf [Accessed November 14, 2014]).

Niida, A., et al., 2004. DKK1, a negative regulator of Wnt signaling, is a target of the beta-catenin/TCF pathway. Oncogene 23 (52), 8520-8526 (Available at: http://dx. doi.org/10.1038/sj.onc.1207892 [Accessed August 12, 2014]).

Oginuma, M., et al., 2010. The oscillation of Notch activation, but not its boundary, is required for somite border formation and rostral-caudal patterning within a somite. Development 137 (9), 1515-1522 (Available at: http://dev.biologists.org/content/ 137/9/1515.full [Accessed April 7, 2015]).

Palmeirim, I., et al., 1997. Avian hairy gene expression identifies a molecular clock linked to vertebrate segmentation and somitogenesis. Cell 91 (5), 639-648 (Available at: http://www.sciencedirect.com/science/article/pii/S0092867400804511 [Accessed March 23, 2014]).

Pan, D., 2010. The hippo signaling pathway in development and cancer. Dev. Cell 19 (4) 491-505 (Available at: http://www.cell.com/article/S1534580710004296/fulltext [Accessed May 8, 2014])

Shimojo, H., Ohtsuka, T., Kageyama, R., 2008. Oscillations in notch signaling regulate maintenance of neural progenitors. Neuron 58 (1), 52-64 (Available at: http://www sciencedirect.com/science/article/pii/S0896627308001669 [Accessed July 14, 2014]).

Sievers, F., et al., 2011. Fast, scalable generation of high-quality protein multiple sequence alignments using Clustal Omega. Mol. Syst. Biol. 7, 539 (Available at: http:// www.pubmedcentral.nih.gov/articlerender.fcgi?artid=3261699\&tool=pmcentrez\& rendertype $=$ abstract $[$ Accessed March 19, 2014])

St John, J.A., et al., 2012. Sequencing three crocodilian genomes to illuminate the evolution of archosaurs and amniotes. Genome Biol. 13 (1), 415 (Available at: http://genomebiology. com/2012/13/1/415 [Accessed March 23, 2014]).

Stadler, C., et al., 2012. Systematic validation of antibody binding and protein subcellular localization using siRNA and confocal microscopy. J. Proteome 75 (7), 2236-2251 (Available at: http://www.sciencedirect.com/science/article/pii/ S187439191200070X [Accessed March 23, 2014]).

Tax, F.E., Yeargers, J.J., Thomas, J.H., 1994. Sequence of C. elegans lag-2 reveals a cell-signalling domain shared with Delta and Serrate of Drosophila. Nature 368 (6467), 150-154 (Available at: http://dx.doi.org/10.1038/368150a0 [Accessed October 2, 2014]).

Theiler, K., 1989. The house mouse: atlas of embryonic development. SpringerVerlag, New York. 
Vassilev, A., et al., 2001. TEAD/TEF transcription factors utilize the activation domain of YAP65, a Src/Yes-associated protein localized in the cytoplasm. Genes Dev. 15 (10) 1229-1241 (Available at: http://genesdev.cshlp.org/content/15/10/1229.full [Accessed April 29, 2014]).
Wysocki, K., Conley, Y., Wenzel, S., 2014. Epigenome Variation in Severe Asthma. Biol. Res. Nurs. (Available at: http://brn.sagepub.com/content/early/2014/10/ 03/1099800414553463.abstract [Accessed October 31, 2014], p.109980041 4553463-). 